Fruehmann, R.K., Dulieu-Barton, J.M. Quinn S. and Tyler, J.P., "The use of a lock-in amplifier to apply digital image correlation to cyclically loaded components", Optics and Lasers in Engineering, 68, 2015, Pages 149-159.

\title{
The use of a lock-in amplifier to apply digital image correlation to cyclically loaded components
}

\author{
R. K. Fruehmann ${ }^{1, \mathrm{a}}$, J. M. Dulieu-Barton ${ }^{1, \mathrm{~b}}$, S. Quinn ${ }^{1, \mathrm{c}}$, J. P. Tyler ${ }^{2, \mathrm{~d}}$ \\ ${ }^{1}$ University of Southampton, Faculty of Engineering and the Environment, SO17 1BJ \\ ${ }^{2}$ Enabling Process Technologies Ltd., Bristol, BS20 8ET \\ ${ }^{a}$ rkf@soton.ac.uk, janice@soton.ac.uk, ${ }^{\mathrm{c}}$ S.quinn@soton.ac.uk, ${ }^{\mathrm{d}}$ philip.tyler@eptworld.com
}

\begin{abstract}
An approach for processing strain data obtained from Digital Image Correlation (DIC) that can be used directly on components subject to cyclic loading, such as in fatigue testing, is described. A key challenge addressed herein is obtaining an accurate and precise surface strain map without recourse to expensive high speed cameras to capture data to map the load cycle. The basis of the approach is the application of a lock-in amplifier to the strain data obtained from images taken from low-cost low-frame rate cameras. The technique enables images to be captured throughout a fatigue test and hence evaluate the effect of any damage on the strain field, without interrupting the test. The methodology is assessed using an aluminium disc in diametric compression. It is demonstrated that the approach enables accurate strain maps to be obtained using a range of loading frequencies that are greater than the camera frame rate. A realistic example application of the technique on a fatigue test with an evolving crack in a T-shaped specimen is presented.
\end{abstract}

Keywords: DIC, fatigue testing, damage inspection, lock-in amplifier

\section{Introduction}

The use of full-field optical techniques has grown over the past two decades due to the progress in digital camera performance and increased computational power. The full-field nature of optical techniques enables areal inspection of strains making them ideally suited for identifying strain concentrations and / or damage. Digital image correlation (DIC) [1] has become the most popular of these imaging based techniques because of its versatility and robustness. The technique is based on the use of a correlation algorithm to track surface patterns (typically a black and white painted random speckle [2]) and hence obtain the displacements and strains that occur during loading. To perform a correlation requires a minimum of two images, an initial reference image and a second image taken after some deformation has occurred. Recent reviews [1, 3, 4] provide full details of the status of the technique and its application range. There have been numerous studies of the measurement errors in DIC, e.g. [5], many of these emanate from the application of the correlation 
algorithm and the sub-pixel interpolation approaches, as well as camera noise, sensor construction, localised heating due to lighting to obtain sufficient contrast and parasitic effects from the loading.

Most of the application of DIC has been carried out on structures under static load; a recent application demonstrating the spatial and strain resolution that can be achieved is described in [6]. In the present paper the application of DIC to a dynamic test involving cyclic loading is investigated. A motivation for the work is in the application to fatigue tests which currently rely predominantly on single point measurements using strain gauges and global measures such as actuator displacement and load, which risk missing critical strain concentrations in unexpected locations. As fatigue tests are very time consuming and accordingly expensive, a full-field technique such as DIC, that would elicit more information about the structural behaviour and enable more efficient testing approaches, is highly desirable. This presents two main challenges: 1) it is preferable not to interrupt the fatigue test for the purpose of obtaining a measurement, as this is labour intensive and introduces additional parameters to the test due to the extended dwell time at high load, and 2) both a high strain and spatial resolution are required to identify damage initiation sites at an early stage. It was therefore deemed as crucial to establish an automated means capturing images suitable for DIC, without pausing the fatigue test and without any user interaction.

Several approaches have been proposed to enable DIC for dynamic tests, most of which employ some method of synchronisation to obtain images at the maximum and minimum loads, thereby simulating a static test. This can take the form of stroboscopic lighting synchronised to the loading [7] or directly synchronising the image trigger to the loading cycle [8]. However, such synchronisation introduces additional complexity and cost to the test set-up and does not provide any improvement in terms of strain or spatial resolution compared to the standard static approach. An alternative approach is to make use of additional knowledge about the temporal variation of the strains, e.g. knowledge of the periodic nature of the fatigue loading. By collecting a series of images over a number of loading cycles it is possible to describe the strain at each point on the specimen as a function of time; the strain changes of interest occur at a known frequency, and all other frequency components in the calculated strain can be considered measurement noise. An appropriate signal processing approach is then required to assess the amplitude of the deformations. In this case the image capture does not need to be synchronised to the loading, thereby simplifying the setup. Such an approach has been tried in [9] in which a discrete Fourier transform (DFT) was used to obtain the amplitude of the displacement fields from which the stress intensity factors were calculated. The DFT approach enables a spectrum of frequencies to be assessed. However, many fatigue tests are conducted at a single frequency and the aim of the processing is to eliminate noise in the measurement at all other frequencies. For this application, a well established method is the lock-in 
Fruehmann, R.K., Dulieu-Barton, J.M. Quinn S. and Tyler, J.P., "The use of a lock-in amplifier to apply digital image correlation to cyclically loaded components”, Optics and Lasers in Engineering, 68, 2015, Pages 149-159.

amplifier as used in thermoelastic stress analysis (TSA) [10]. TSA is a full-field stress analysis technique in which the lock-in amplifier is used to filter surface temperature measurements to identify a very small temperature change related to the thermoelastic response in a material. The lock-in amplifier is able to provide temperature resolutions of the order of a few $\mathrm{mK}$ within a measurement noise of the order of $10 \mathrm{~s}$ of $\mathrm{mK}$. Since TSA requires the specimen to be subjected to cyclic loading, the technique naturally lends itself to fatigue testing and there are numerous examples where the technique has been used to monitor damage progression in composites and crack growth in metals e.g. $[11,12]$. However, TSA provides data that is related only to the change in the sum of the principal stresses, i.e. it does not provide individual stress or strain components. The great increase in thermal resolution achieved using the lock-in amplifier in TSA inspired the idea to use this approach for DIC. Since DIC provides individual strain components, it presents a complementary approach to TSA and the two techniques could conceivably be applied together, with no special requirements for synchronisation.

To demonstrate the application of DIC to components undergoing cyclic loading and highlight the challenges and potential benefits, a Brazilian disc test is used. This test provides a biaxial strain field with a range of strains and strain gradients. Hence it is ideal for demonstrating both the spatial and strain resolution of the proposed approach. The availability of an analytical solution provides a good basis for comparison, and the test can be conducted both in a static and a dynamic manner for a quantitative comparison to the current usual approach that employs just two images to obtain a strain field. An example application where the strain field in the neighbourhood of a growing fatigue crack is monitored and analysed.

\section{Overview of signal filtering using lock-in approaches}

The lock-in amplifier is an established tool for obtaining the amplitude and phase of small sinusoidal signals embedded within a noisy signal $[13,14,15]$. In this work, the lock-in amplifier is implemented as a software tool to filter a measurement signal. In brief, the lock-in amplifier multiplies an input (measurement) signal by a reference signal and integrates the result over a number of periods. This produces a DC level proportional to the amplitude of only that component of the input signal at the same frequency as the reference signal [10]. The reference signal is a single frequency signal with very low noise content. The lock-in amplifier can be considered a band pass filter that discards all signal components other than those synchronised with the reference signal. The noise rejection is improved by increasing the duration of the recording relative to the 
reference signal period; the longer recorded signal the narrower the band pass and the better the noise rejection. Where the signal does not vary over time, it is therefore best to include as many periods in the analysis as practical, although in practice there is a threshold beyond which no discernable benefit is achieved.

The lock-in amplifier is phase sensitive, i.e. two signals of identical frequency but $90^{\circ}$ out of phase will output an amplitude of zero, requiring the reference and measurement signals to be exactly in phase to obtain an accurate measure of the amplitude. Hence a two phase lock-in amplifier is applied that uses not only the reference signal, but also its quadrature to output an $\mathrm{X}$ and a $\mathrm{Y}$ component from which the amplitude and phase angle between the input and reference signals can be obtained:

$$
\begin{aligned}
& X=\frac{1}{N / 2} \sum_{i=1}^{N} f\left(t_{i}\right) \sin \left(\omega t_{i}+\Phi\right) \\
& Y=\frac{1}{N / 2} \sum_{i=1}^{N} f\left(t_{i}\right) \cos \left(\omega t_{i}+\Phi\right) \\
& A=\sqrt{X^{2}+Y^{2}} \\
& \Phi=\tan ^{-1}(Y / X)
\end{aligned}
$$

where $N$ is the number of samples, $i$ is the sample number, $f\left(t_{i}\right)$ is the input signal as a function of time $t, \sin \left(\omega t_{i}+\Phi\right)$ is the reference signal with frequency $\omega$ and phase $\Phi, \cos \left(\omega t_{i}+\Phi\right)$ is the quadrature of the reference signal and $A$ is the amplitude.

To apply the lock-in amplifier process in an experiment where white light images are collected from a specimen undergoing cyclic loading, it is firstly necessary to use DIC to obtain the displacement and hence the strain field relative to the first image. For each image correlation the strain is determined for each interrogation cell in a given image as a function of time. A reference signal, typically from a test machine load cell, is recorded so that the quadrature of the reference signal (i.e. $90^{\circ}$ phase shifted) can be calculated. Equations (1) through (4) can then be applied to the strain signal at each interrogation cell, giving a map of the amplitude and phase of the strain change over the component. Figure 1 shows a flow chart of the procedure for lock-in DIC.

Note that the reference signal must have a mean of zero and an amplitude of 1 in order not to scale the results, as per equations (1) and (2). This was done by generating a normalised reference signal according to: 
Fruehmann, R.K., Dulieu-Barton, J.M. Quinn S. and Tyler, J.P., “The use of a lock-in amplifier to apply digital image correlation to cyclically loaded components", Optics and Lasers in Engineering, 68, 2015, Pages 149-159.

$r_{\text {norm }}\left(t_{i}\right)=\left(r\left(t_{i}\right)-\bar{r}\right) / A_{r}$

where $r\left(t_{i}\right)$ is the reference signal, $r_{\text {norm }}$ is the normalised reference signal, $\bar{r}$ is the mean of the reference signal and $A_{r}$ is the amplitude of the reference signal obtained using a fast Fourier transform. The quadrature was obtained as the imaginary part of a Hilbert transform of the normalised reference signal, using the Matlab command 'hilbert'.

The analysis makes several assumptions about the signal that do not hold true for finite, discretely sampled signals. Therefore a number of conditions need to be carefully considered. For example, the length of any recorded signal is finite, limited by hardware (e.g. computer storage space) or the test conditions (e.g. in a fatigue test the number of load cycles over which the conditions can be considered 'steady state'). The signal is therefore 'truncated'; underlying the analysis however, is the assumption that the signal is infinite. To maintain validity, it is therefore necessary to impose the condition of periodicity on the recorded signal, i.e. the recorded signal must sample an integer number of periods. If not, this results in what is termed 'spectral leakage' and consequently an underestimate of the signal amplitude [16]. However, in the case of the lock-in amplifier where the frequency of interest is well defined by the reference signal; by truncating the signal to meet the periodicity condition for the particular frequency of interest, spectral leakage and other aliasing effects can be minimised [17].

Shannon's sampling theorem [18] requires that the sampling frequency is greater than two times the maximum frequency contained in the measurement signal (the Nyquist or critical frequency). Since the sampling frequency is limited, this leads to the requirement that the measurement signal is band limited, i.e. the maximum frequency contained within the signal must not exceed half the sampling frequency. However, real signals are rarely band limited in this way, and hence this can lead to aliasing. For example, consider a $0.2 \mathrm{~Hz}$ signal with a noise content of frequency $3.2 \mathrm{~Hz}$, if this is sampled at $1 \mathrm{~Hz}$ the output of the lock-in amplifier will be the sum of the two amplitudes, giving a false estimate of the $0.2 \mathrm{~Hz}$ signal amplitude. This only applies for specific combinations of input, reference and sampling frequencies and will generally not pose a problem when using the lock-in amplifier. However, the fact that the lock-in amplifier can respond to frequencies greater than the sampling frequency also presents an opportunity.

One of the challenges in DIC is that high resolution cameras tend to have very low frame rates by signal processing standards. Also, the size of the data tends to be very large, since each point on the time axis is an image comprising several million points. Hence it is not feasible to record very long signals. A typical mono-chrome 12 bit camera as used in DIC will have a maximum frame rate of 
10's of Hz, and if more than one camera is used simultaneously with a standard desktop PC, this may quickly reduce to 1's of Hz. A typical fatigue test will run at 1's to 10's of Hz, and hence the Nyquist condition is difficult to meet. The solution to opt for high speed cameras is expensive and typically linked to a much reduced image spatial resolution. Therefore, it is preferable to use standard high resolution, low speed cameras, and make use of the fact that it is possible to analyse signals with frequencies greater than the sampling frequency. When undersampling a signal, it is important to remember that the lock-in algorithm is not generally valid, and that the sampling and reference signals need to be carefully selected to avoid aliasing problems. Since the lock-in algorithm cannot know the actual signal frequency, the discrete signal is treated as if it had a lower frequency. This is shown graphically in Figure 2, using an example from the following section where a $2 \mathrm{~Hz}$ sampling frequency is used to analyse a $7.1 \mathrm{~Hz}$ signal. The actual signal has a frequency of $7.1 \mathrm{~Hz}$. When sampled at $2 \mathrm{~Hz}$, this gives the discrete signal represented by the crosses. These sampling points coincide with a signal at $0.9 \mathrm{~Hz}$. The lock-in algorithm therefore treats the measured signal as though it had a frequency of $0.9 \mathrm{~Hz}$. Hence, the duration of the sampling has to be selected so that an integer number of $0.9 \mathrm{~Hz}$ periods are included in the sampling period, i.e. multiples of 10 seconds, as shown in Figure 2. Another important consideration is to avoid loading frequencies that are exact integer multiples of Nyquist frequency (i.e. half the sampling frequency) as this would result in the measurement signal being sampled at only one or two points on the cycle. If the sampling points happen to coincide with a peak and a trough, then an exact estimate of the amplitude would be obtained. However, if the points coincide with the zero crossings, then the measured signal would be a flat line and the calculated amplitude would accordingly be zero.

Having selected a suitable combination of sampling and loading frequencies, to minimise image blurring it is necessary to ensure that the camera exposure time is short relative to one period of the loading cycle. Image blurring will be worst at the zero crossing, where motion is fastest. The maximum acceptable exposure time is dependent on the size of the motion relative to the camera resolution. For example, for an image resolution of $0.049 \mathrm{~mm} / \mathrm{px}$ and a displacement amplitude of $0.07 \mathrm{~mm}$, taking $10 \%$ of the displacement amplitude as the maximum amount of blurring acceptable leads to an edge blurring effect of $14 \%$ of the pixel size. For a 3 x 3 pixel speckle, the motion blurring effect would be $3 \%$ of the speckle size. By comparison, the blurring effect due to the size of the pixel relative to the size of the speckle would be of the order of $30 \%$, and so the motion blurring could be considered negligible. For a loading frequency of $7.1 \mathrm{~Hz}$ (as per the above example) the period is $0.14 \mathrm{~s}$ and therefore the exposure time must be less than $4.2 \mathrm{~ms}$ to meet the $10 \%$ criterion. 
Fruehmann, R.K., Dulieu-Barton, J.M. Quinn S. and Tyler, J.P., "The use of a lock-in amplifier to apply digital image correlation to cyclically loaded components”, Optics and Lasers in Engineering, 68, 2015, Pages 149-159.

In setting up the experiment to demonstrate the application of the lock-in method to DIC, a range of loading frequencies were used starting with a static test (the standard approach used as a benchmark to compare performance), $0.75 \mathrm{~Hz}$ (compliant with Shannon's sampling theorem), $7.1 \mathrm{~Hz}$ and 21.1 $\mathrm{Hz}$ (undersampled). The camera frame rate $(2 \mathrm{~Hz})$ and the length of the recording (250 images) were kept the same throughout. The loading frequencies and exposure times were selected according to the sampling parameters. This approach was chosen so to give a consistent set of data to enable an evaluation of the method. It is recognised that in a fatigue test it is more likely that the loading frequency is predetermined, and the recording frequency and length would be adjusted accordingly.

\section{Demonstration on a Brazilian disc}

The camera used in this work was a Manta G504-B from Allied Vision with a 2452 x 2056 sensor with $3.45 \mu \mathrm{m}$ pitch, an 8 bit grey scale, $40 \mathrm{~mm}$ Nikon lens and a frame rate of $2 \mathrm{~Hz}$. The test machine used was an Instron 8820 load frame with a $100 \mathrm{kN}$ servo-hydraulic actuator and corresponding $100 \mathrm{kN}$ dynamic load cell. The load cell output was used to provide the reference signal for the lock-in processing. The DIC was performed with the commercial software DaVis 8.1.0 from LaVision. The software uses a least squares approach to calculate displacements. Strains are calculated from the displacement field using a central differencing scheme, i.e. the strain value at any given cell is calculated using the displacements from the adjacent cells.

The specimen used was an $80 \mathrm{~mm}$ diameter, $6 \mathrm{~mm}$ thick 8061-T6 aluminium disc, loaded in compression between two flat mild steel platens, i.e. the Brazilian disc set up [19]. For the static test, an initial load of $-0.5 \mathrm{kN}$ was applied to hold the disc between the platens, and a peak load of $9.5 \mathrm{kN}$. For the dynamic test a sinusoidal load with a mean of $-5 \mathrm{kN}$, an amplitude of $\pm 4.5 \mathrm{kN}$ and a range of loading frequencies was applied, giving the same load range as for the static case. A quantitative assessment of the DIC strain fields was made by comparison to the analytical solution [20]. The strain fields can then be calculated using the elastic material properties: the Young's modulus (70 GPa), shear modulus (26 GPa) and Poisson's ratio $(0.33)$.

A matt white background was sprayed on the specimen surface over which a matt black speckle was applied using a RS Matt black aerosol spray paint. The aim was to generate a relatively high density of small speckles, approximately 3 x 3 image pixels in size. It can be seen in Figure 3 that there is a large range of speckle sizes, but the larger speckles are fairly few. The majority of speckles lie in the $2 \times 2$ to $4 \times 4$ pixel size range. The insert shows a magnification of a sample area of the disc with a 
representative $21 \times 21$ pixel grid with 10 pixel overlap superimposed to show the relative size of the speckles and correlation cells. It should be noted that a circular mask was used to select only such cells as lay completely within the boundaries of the disc. There is also a small region in the upper right quadrant of the disc where the black paint has formed a more uniform coating leading to a reduction in contrast. It was decided to retain the coating to ascertain the effect of the more uniform area on the data; this will be discussed later in the paper. The camera was set up $80 \mathrm{~cm}$ from the specimen, giving a resolution of $0.049 \mathrm{~mm} /$ pixel. The integration time (exposure) was set to 1100 $\mu$ s to minimise image blurring due to motion. Lighting was provided by an LED array.

The first step was to select a suitable interrogation cell size for comparing the static and the dynamic tests. This was done using data from the oversampled dynamic case $(0.75 \mathrm{~Hz}$ loading frequency). Four interrogation cell sizes were chosen, 41 x 41 pixels, 31 x 31 pixels, 21 x 21 pixels and $11 \times 11$ pixels, with a 50\% overlap in all cases. A comparison between the strain obtained using different interrogation cell sizes is presented in Figure 4. The y-direction strains, $\varepsilon_{\mathrm{y}}$, along the vertical diameter of the disc are presented because these cover a large range of strains, from the largest strains at the load introduction points to the relatively uniform low strain region towards the centre of the disc. The $41 \times 41$ and $31 \times 31$ pixel subsets provide very smooth curves, indicating a low noise threshold. By comparison, the $21 \times 21$ pixel subset size starts to display a significant amount of noise at strain magnitudes less than $10^{-3}$ in the central part of the disc, but still has a good resolution of the strain gradients approaching the load introduction points. In the data from the smallest subset size tested, $11 \times 11$ pixels, the noise is so large that trends in the strain field cannot be discerned. The 21 × 21 pixel subset size therefore represents the lower limit at which a reasonable strain resolution can be achieved with the particular combination of speckle size, speckle distribution, detector resolution and magnification used in this experiment. Therefore it was considered the best subset size to illustrate differences in performance between the standard static approach and the dynamic approach using the lock-in amplifier.

The static test was conducted first. Figure 5 a) shows the y-direction displacement field, with a maximum displacement of approximately $0.15 \mathrm{~mm}$ at the lower end where the actuator was located. In the $0.75 \mathrm{~Hz}$ dynamic test, conducted second, a reduction in the total y-displacement (Figure $5 \mathrm{~b}$ )) can be observed. The range of displacements across the disc is still the same; the small difference in the overall displacement occurs only at the very ends where a small amount of plastic deformation has occurred after the first load application, This plastic deformation leads to a reduction in the peak strains at the load introduction points as the load is spread over a small area. This difference is apparent later in the comparison of the strain fields very near the edge of the disc. Another consideration with compression testing is the tendency for the loading frame to displace laterally. 
Fruehmann, R.K., Dulieu-Barton, J.M. Quinn S. and Tyler, J.P., "The use of a lock-in amplifier to apply digital image correlation to cyclically loaded components”, Optics and Lasers in Engineering, 68, 2015, Pages 149-159.

This is exaggerated in servo-hydraulic test machines (by comparison to screw driven test machines) due to the tolerances at the actuator seals. The x-direction displacements in Figure $5 \mathrm{c}$ ) and d) show a small clockwise rotation of the specimen resulting from a lateral motion of up to $0.01 \mathrm{~mm}$. An out-of-plane displacement of a similar magnitude can be expected. At the stand-off distance of 800 $\mathrm{mm}$, the small change in magnification due to an out-of-plane displacement of $0.01 \mathrm{~mm}$ would lead to a parasitic strain of only $10^{-5}$ which can be considered negligible for this test as it is two orders of magnitude less than the strain resolution.

To provide a benchmark, a strain field was obtained by correlating between only two images, one taken in the reference load state, the second taken under load. However, a significant improvement can be achieved by collecting several images at each load state. During the test 11 images were obtained of the specimen in the reference load state $(-0.5 \mathrm{kN})$, and a further 11 images were obtained at the maximum compressive load $(-9.5 \mathrm{kN})$. There are two approaches to averaging in this data, a pair of images can be generated where each image is the average of the 11 images taken at each load step, and the strain is calculated from the resulting pair of averaged images [21]. Alternatively, a strain field can be obtained for each combination of image pairs, and an average of the resulting strain fields can be obtained. For two sets of 11 images there are 121 combinations of reference and deformed image pairs. Accordingly, 121 static strain fields were obtained, one for each combination of image pairs. To provide a fair comparison with the proposed method using the lock-in algorithm, Figure 6 (showing the y-strains, $\varepsilon_{y}$, i.e. parallel to the loading direction) is divided into 4 quadrants, each with a different level of averaging of strain fields. The top left quadrant is the strain field from a single image pair and represents the standard test approach. The top right is the mean of 5 strain fields, the bottom left is the mean of 25 strain fields and the bottom right is the mean of all 121 strain fields. The improvement in strain resolution can clearly be seen as a reduction in the strain image noise. This approach allows an improvement of strain resolution without impacting on the spatial resolution of the data. In a way analogous to the lock-in approach, knowledge of the 'temporal' distribution of the strains is used to improve the data (i.e. the strain is known to be constant over time, with a step change between only two states).

Figure 7 (also showing $\varepsilon_{\mathrm{y}}$ ) is similarly broken into quadrants to compare the static test mean of 121 strain fields (top left quadrant) to three cases employing the dynamic load and lock-in algorithm. The first of the dynamic test cases (top right quadrant in Figure 7) had a loading frequency of 0.75 Hz. With a sampling frequency of $2 \mathrm{~Hz}$, this complies with the Nyquist condition of sampling a greater than twice the signal frequency; of the 250 recorded strain fields, 248 were used in the lockin processing, covering an exact 31 loading cycles to meet the truncation requirements. The other 
two dynamic cases demonstrate that it is possible to obtain an accurate result outside the Nyquist condition, sampled at $2 \mathrm{~Hz}$ as in the first case, but loaded at $7.1 \mathrm{~Hz}$ (bottom left quadrant in Figure 7) and $21.1 \mathrm{~Hz}$ (bottom right quadrant in Figure 7). An exact integer number of cycles were processed to meet the truncation requirement. In the image data, very little difference can be discerned between the four cases. Figure 8 shows the y-strain values along the vertical diameter of the disc including the theoretical strains for comparison. The standard approach using only one image pair shows the largest scatter, especially in the region where the strains are low. The scatter in the low strain regions for the dynamic tests processed using the lock-in algorithm is generally less when compared to the static tests. Towards the load introduction points at the edge of the disc the static tests appear to capture the higher strains better than the dynamic tests. This can be partially explained by the local plasticity during the first load application discussed earlier. At the loading points the theoretical strain tends towards infinity, and therefore ceases to be a useful baseline for comparison. However, along the horizontal diameter, the strains tend towards zero at the edges. This provides a good comparison of the resolution of the method at low strain levels, shown in Figure 9. The approach with only a single image pair completely fails to capture the strain distribution along the horizontal axis, and a larger interrogation cell size (at the expense of spatial resolution) would be necessary to provide useful strain values.

To demonstrate how positive and negative strains are identified, a plot of $\varepsilon_{\mathrm{x}}$, and the shear strains, $\varepsilon_{\mathrm{xy}}$, is provided in Figure $10 \mathrm{a}$ ) and b), similarly divided into quadrants showing the static, $0.75 \mathrm{~Hz}$, 7.1 Hz and $21.1 \mathrm{~Hz}$ results, ordered top left to bottom right. To convert the strain amplitude values from the dynamic data into positive and negative strains, the phase data was used; a region undergoing compression will be $180^{\circ}$ out of phase with a region in tension. The phase data were converted into a binary image (as shown for the $0.75 \mathrm{~Hz}$ tests in Figure $10 \mathrm{c}$ ) and d)) with values of +1 for a tensile phase and -1 for a compressive phase. This step is not required for the static test.

A comparison of the accuracy of the static and the dynamic tests, is visualised in Figure 11 as the difference between theory and experiment is shown for all three strain components. The images in Figure 11 clearly show that the error is fairly constant over the majority of the specimen, only significantly increasing close to the load introduction points due to the localised plasticity. In the top right quadrant there is a region with increased noise, highlighted by a red circle in Figure $11 \mathrm{c})$. This corresponds with an area where the black paint forms a more uniform layer leading to a reduction in contrast (see also Figure 3). This demonstrates very well the effect of a reduction in contrast which results in a loss in accuracy in the strain calculation.

The reason for expressing the error in absolute terms rather than as a ratio is that when normalising 
Fruehmann, R.K., Dulieu-Barton, J.M. Quinn S. and Tyler, J.P., "The use of a lock-in amplifier to apply digital image correlation to cyclically loaded components”, Optics and Lasers in Engineering, 68, 2015, Pages 149-159.

against a strain value close to zero near the specimen edges, even a small error results in very large percentage error. Since the method has a precision threshold, the regions where the actual strains are very small are exaggerated. To provide a more quantitative comparison, the mean and standard deviation of the errors is presented in Table 1 considering only the strains within a radius of $20 \mathrm{~mm}$ from the centre of the specimen. The relatively large standard deviations in the $\varepsilon_{\mathrm{x}}$ data are due to noise in the phase data leading to incorrect assessment of compressive / tensile strains, leading to a greater than $100 \%$ error when compared to the theoretical value.

Table 1: Strain error in the central part of the disc.

\begin{tabular}{|l|l|l|l|}
\hline & $\begin{array}{l}\text { Mean error in } \varepsilon_{\mathrm{y}} \\
\left(\mathrm{x10}^{-4}\right)\end{array}$ & $\begin{array}{l}\text { Mean error in } \varepsilon_{\mathrm{x}} \\
\left(\mathrm{x10}^{-4}\right)\end{array}$ & $\begin{array}{l}\text { Mean error in } \varepsilon_{\mathrm{xy}} \\
\left(\mathrm{x10}^{-4}\right)\end{array}$ \\
\hline $\begin{array}{l}\text { Theory* } \\
\text { (mean strain) }\end{array}$ & -5.11 & 2.83 & $1.23^{* *}$ \\
\hline Static (mean 121) & $2.14 \pm 1.63$ & $1.97 \pm 1.49$ & $1.61 \pm 1.22$ \\
\hline $0.75 \mathrm{~Hz}$ & $1.30 \pm 0.97$ & $1.08 \pm 1.06$ & $1.17 \pm 0.90$ \\
\hline $7.1 \mathrm{~Hz}$ & $1.35 \pm 0.99$ & $0.97 \pm 0.81$ & $1.15 \pm 0.86$ \\
\hline $21.1 \mathrm{~Hz}$ & $1.34 \pm 1.00$ & $1.31 \pm 1.28$ & $1.30 \pm 1.00$ \\
\hline
\end{tabular}

* This is the mean strain over the central $20 \mathrm{~mm}$ radius area.

** This is the mean of the absolute value of the strains within the central $20 \mathbf{~ m m}$ radius.

\section{Application to a fatigue test}

The specimen used for the fatigue test is a T-shaped plate, gripped in the jaws of the test machine on the vertical surface, and supported by two pins inserted through the transverse 'arms' (as shown in Figure 12). The specimen geometry was designed as part of another project studying the material properties in welded joints, and represents a 2D slice through a T-joint. The work described in the present paper is aimed at assessing the ability to study the propagation of a crack using the DIC in conjunction with lock-in algorithm to enable automated monitoring of crack length during a fatigue test. The specimen was cut from a $5 \mathrm{~mm}$ thick sheet of $316 \mathrm{~L}$ stainless steel and had a $10 \times 0.5 \mathrm{~mm}$ cut introduced at the edge of change in cross-section (as shown in Figure 12) to initiate the controlled growth of a fatigue crack.

To accelerate the fatigue process, the test was run with a $20 \mathrm{~Hz}$ loading frequency. The acquisition of white light images was set to $2.56 \mathrm{~Hz}$ and 300 images were obtained for each strain calculation. Hence the acquisition of the white light images spanned approximately 2000 load cycles. TSA was therefore used to monitor the back-side of the specimen to assess if the scene could be considered stationary over the course of the recording. Since the infrared detector used for the TSA operates at $383 \mathrm{~Hz}$, each recording (comprising 1200 images) spans only 60 cycles, and because the lock-in 
analysis is applied directly to the infrared data, the data analysis only takes a matter of seconds. Hence images of the amplitude of the thermoelastic response (i.e. the sum of the principal stresses) could be obtained and compared in real time during the course of the fatigue testing. The spatial resolution of the TSA data was 2.93 pixels/ mm. A pair of temperature amplitude images $(\Delta T)$ obtained at 100,000 and 102,000 cycles are shown in Figure 13 a) and b) respectively. Figure 13 c) shows the difference between them from which it is clear that the scene has changed over the course of the 2000 load cycles. On account of this difference appearing in the TSA data between start and finish of the set of images acquired for the DIC, the test was stopped after 102,000 cycles as the assumption of a static scene could no longer be applied.

To provide automated white light image acquisition for the DIC, the camera used in this part of the work was an Imager E-lite from LaVision. The camera has a 2491 x 2093 pixel sensor with a pixel pitch of $3.45 \mu \mathrm{m}$. A stand-off distance of $630 \mathrm{~mm}$ was used to provide a view of the complete specimen, and gave an image resolution of 28.4 pixels $/ \mathrm{mm}$. A similar speckle pattern was applied as for the Brazillian disc although variation due to the random nature of the process led to a slightly denser pattern with slightly finer speckles, shown in Figure 14. strain The frame rate was set to 2.56 $\mathrm{Hz}$ with an exposure of $1500 \mu$ s and 300 frames were collected for each calculation. The specimen was loaded at $20 \mathrm{~Hz}$, with an amplitude of $1 \mathrm{kN}$ about a mean load of $2 \mathrm{kN}$. Each DIC analysis therefore spans just over 2000 cycles.

The global strain field was assessed with a subset size of $21 \times 21$ pixels with a step size of 5 px. In the $\varepsilon_{\mathrm{x}}$ data shown in Figure 15 at 0 and 100,000 cycles, the strain concentrations at the change in cross-section and at the end of the crack are clearly visible. Spurious strains are generated at the crack due to the discontinuity and associated large displacement across the crack. Also clearly visible is the increase in compressive strain along the edge opposite the crack. There are two areas on the specimen were a large droplet from the spray can lead to a speckle larger than the $21 \times 21$ pixel subset. These are visible as noise in the strain field in the top left corner of the specimen, and the bottom right, adjacent to the crack. Over the majority of the specimen however, the speckle was uniform in terms of size and distribution.

To assess the change in crack length, the x-displacement fields were used in conjunction with an edge detection algorithm in Matlab using the Sobel method for assessing spatial gradients. This method considers gradients in image intensity based on a 3 x 3 matrix of image points. Edges are identified where the gradient is a maximum. A threshold value can be set to define the sensitivity of the edge detection to avoid spurious measurements resulting from noise in the image. To identify the vertically orientated crack, the horizontal (i.e. x-direction) displacement field was used. Figure 
Fruehmann, R.K., Dulieu-Barton, J.M. Quinn S. and Tyler, J.P., “The use of a lock-in amplifier to apply digital image correlation to cyclically loaded components”, Optics and Lasers in Engineering, 68, 2015, Pages 149-159.

16 shows the $\mathrm{x}$-direction displacement fields at 0 and 100,000 cycles on the left. The corresponding cracks identified using the edge detection algorithm are shown on the right hand side. The threshold value was selected by trial and error, increasing the value until known spurious edges detected within the specimen area were eliminated. The values used ranged from 0.007 to 0.009 . The crack can be seen to grow at an angle to the artificial pre-crack. The crack length was assessed only in the vertical direction by counting the length of the detected edge in strain image pixels (i.e. multiples of 5 raw image pixels) and multiplying by the image scale. The trend in crack length is presented in Figure 17.

For a detailed analysis of the strain field at the crack tip, a higher spatial resolution and lower loading rate would be necessary. However, the results presented herein demonstrate how the technique can be applied to monitor a fairly large area for the presence and growth of a relatively small feature.

\section{Conclusions}

It is demonstrated that the lock-in algorithm can effectively be applied to strain data obtained using DIC.

The low frame rate capability of standard digital cameras does not present an unsurmountable challenge in the application of DIC at high loading rates as an undersampled signal can be analysed accurately.

An improvement in strain resolution compared to a standard static test is achieved using the lock-in algorithm. In particular, the noise threshold is reduced, enabling smaller interrogation cells to be used and thereby improving spatial resolution without recourse to more expensive, high resolution cameras.

The application of the method to fatigue data is demonstrated. While the method provides a high level of strain resolution, the issue of frame rate relative to the rate of change of the observed strain field is shown to be an issue during accelerated fatigue testing (i.e. at high loading frequencies). A lower loading rate as commonly used in fatigue testing would alleviate this issue.

The application of the lock-in algorithm enables DIC data to be readily used in conjunction with TSA data at comparable spatial resolutions.

\section{Acknowledgements}

The authors would like to thank the UK Technology Strategy Board and the EU MATERA+ 
funding bodies for providing the financial support for this work.

\section{References}

[1] M. A. Sutton, J.-J. Orteu, and H. W. Schreier, "Image correlation for shape, motion and deformation measurements," Springer, (2009)

[2] G. Crammond, S.W. Boyd and J.M. Dulieu-Barton, "Speckle pattern quality assessment for digital image correlation," Optics and Lasers in Engineering, 51, pp.1368-1378, (2013)

[3] B. Pan, K. Qian, H. Xie, and A. Asundi, "Two-dimensional digital image correlation for inplane displacement and strain measurement: a review," Measurement Science and Technology, 20, $17 \mathrm{pp},(2009)$

[4] B. Pan, "Recent Progress in Digital Image Correlation," Experimental Mechanics, 51, pp. 1223-1235, (2011)

[5] P. Lava, S. Cooreman, S. Coppieters, M. De Strycker, and D. Debruyne, "Assessment of measuring errors in DIC using deformation fields generated by plastic FEA," Optics and Lasers in Engineering, 47, pp. 747-753, (2009)

[6] G. Crammond, S.W. Boyd and J.M. Dulieu-Barton, "Evaluating the localised throughthickness load transfer and damage initiation in a composite joint using digital image correlation" Composites Part, In Press.

[7] P. Wu and P. Ifju, "Flapping wing structural deformation and thrust correlation study with flexible membrane wings," AIAA Journal, 48, pp. 2111-2122, (2010)

[8] M. Risbet, P. Feissel, T. Roland, D. Brancherie, J-M Roelandt, "Digital Image Correlation technique: application to early fatigue damage detection in stainless steel", Procedia Engineering, 2, pp.2219-2227, (2010)

[9] S.Vanlanduit, J. Vanherzeele, R. Longo and P. Guillaume, "A digital image correlation method for fatigue test experiments", Optics and Lasers in Engineering, 47, pp. 371 - 378, (2009)

[10] N. Harwood and W.M. Cummings, “Theromelastic Stress Analysis,” IOP Publishing, (1991)

[11] T.R. Emery and J.M. Dulieu-Barton, "Thermoelastic stress analysis of damage mechanisms in composite materials," Composites - Part A, 41, 1729-1742, (2010) 
Fruehmann, R.K., Dulieu-Barton, J.M. Quinn S. and Tyler, J.P., “The use of a lock-in amplifier to apply digital image correlation to cyclically loaded components", Optics and Lasers in Engineering, 68, 2015, Pages 149-159.

[12] F.A. Diaz, E.A. Patterson, R.A. Tomlinson and J.R. Yates "Measuring stress intensity factors during fatigue crack growth using thermoelasticity", Fatigue \& Fracture of Engineering Materials and Structures, 27, 571-583, (2004)

[13] P.A. Temple, "An introduction to phase sensitive amplifiers: an inexpensive student instrument," American Journal of Physics, 43, pp.801-807, (1975)

[14] J.H. Scofield, "Frequency-domain description of a lock-in amplifier," American Journal of Physics, 62, pp.129-133, (1975)

[15] J. Gaspar, S.F. Chen, A. Gordillo, M. Hepp, P. Ferreyra and C. Marqués, "Digital lock-in amplifier: study, design and development with a digital signal processor," Microprocessors and Microsystems, 28, pp. 157-162, (2004)

[16] C. Ibarra-Castanedo and X Maldague, "Pulsed phase thermography reviewed," Quantitative Infrared Thermography Journal, 1, pp 47-70, (2004)

[17] M. Unser, "Sampling - 50 years after Shannon," Proceedings of the IEEE, 88, pp. 569-587, (2000)

[18] C.S. Shannon, "Communication in the presence of noise," Proceedings of the IRE, 37, 1021, (1949)

[19] C-S. Chen, E. Pan and B. Amadei, "Determination of deformability and tensile strength of anisotropic rock using Brazilian tests," International Journal of Rock Mechanics and Mining Sciences, 35, pp. 43-61, (1998)

[20] N.I. Muskhelishvili, "Some basic problems of the mathematical theory of elasticity, $2^{\text {nd }}$ English edition", Noordhoff International Publishing, (1977)

[21] C. Devivier, "Damage identification in layered composite plates using kinematic full-field strain measurements," Université de Technologie de Troyes, PhD Thesis, (2012) 
Figures

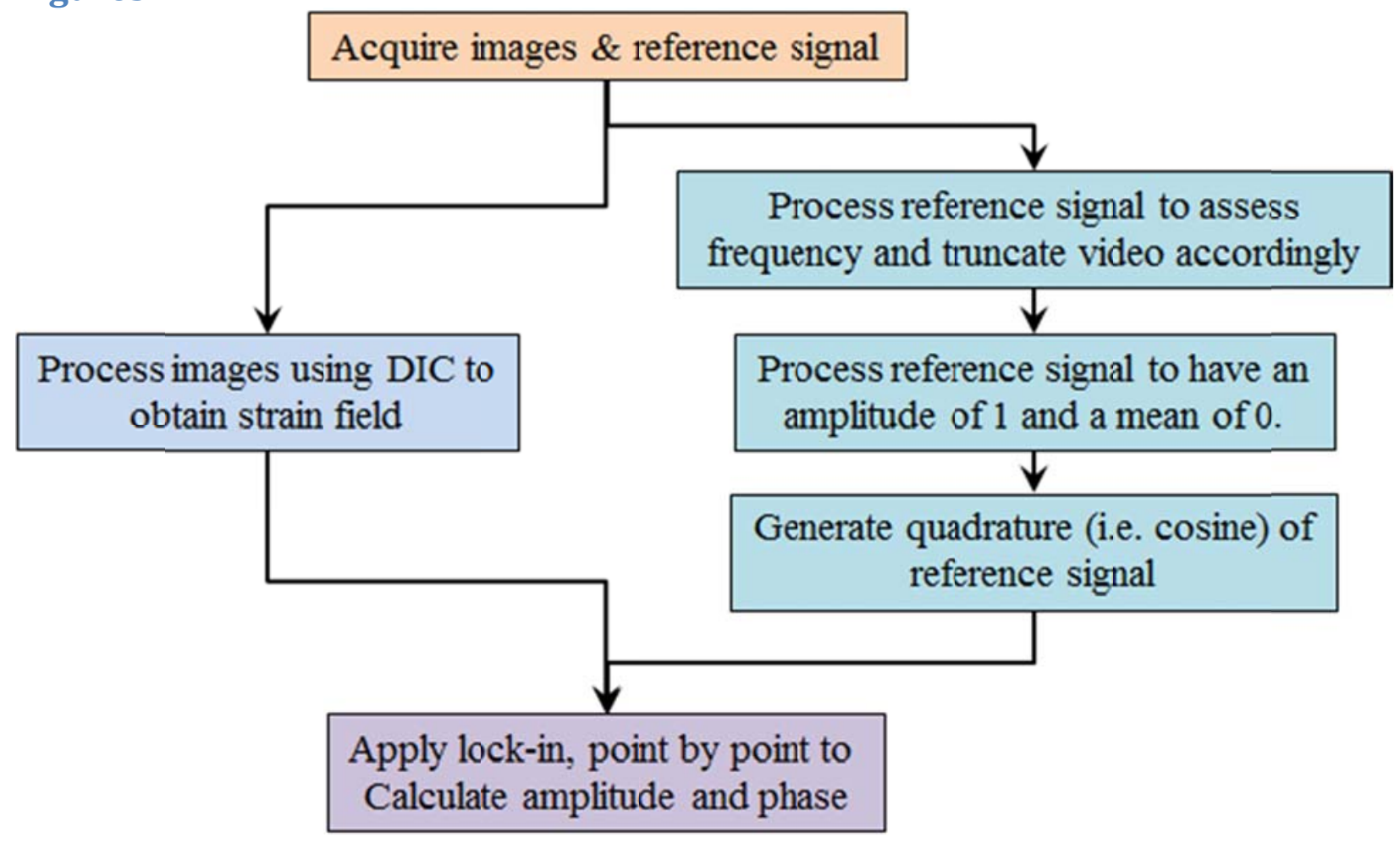

Figure 1: Lock-in processing flow

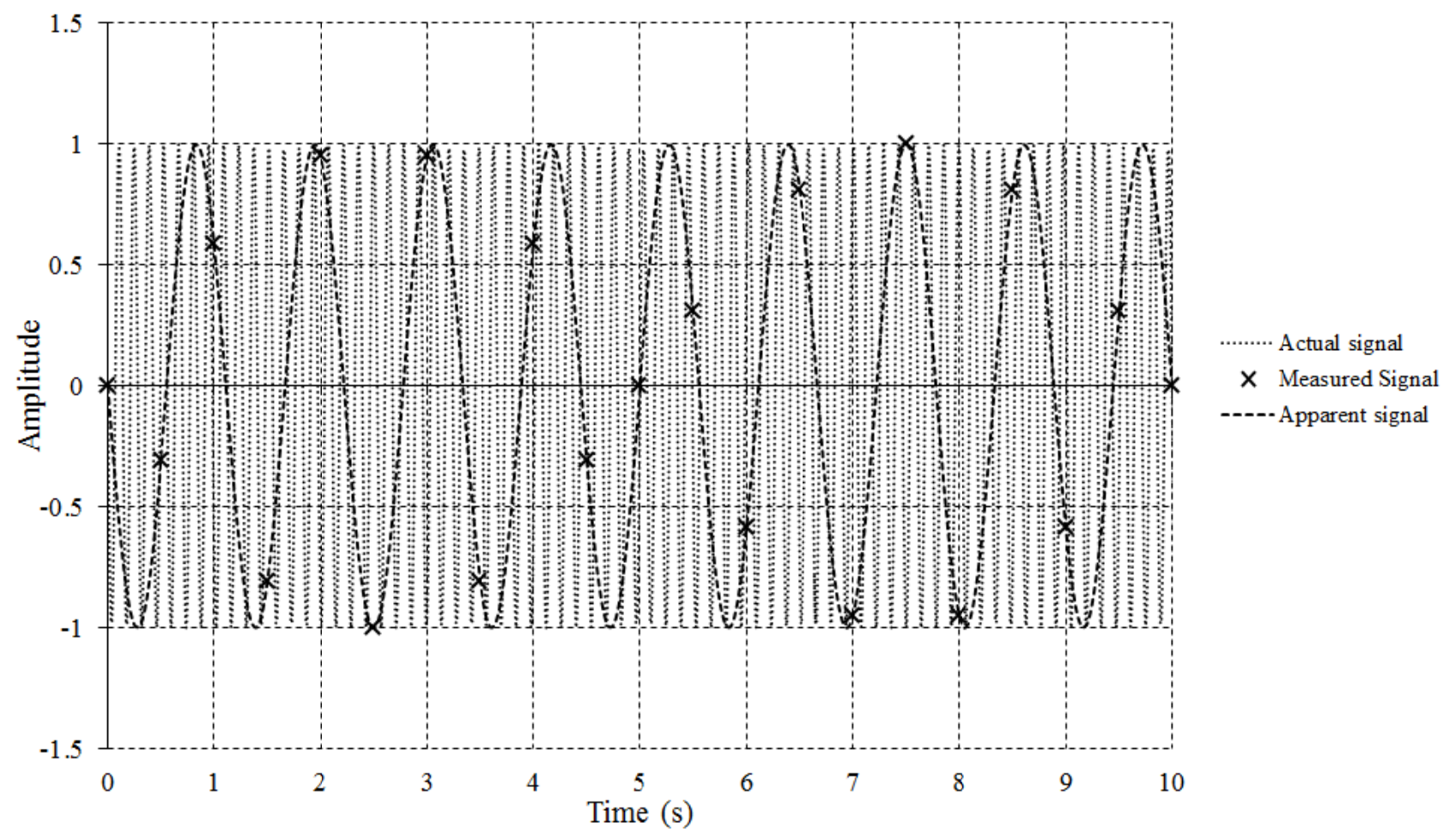

Figure 2: Visualisation of the one complete period of an undersampled signal 


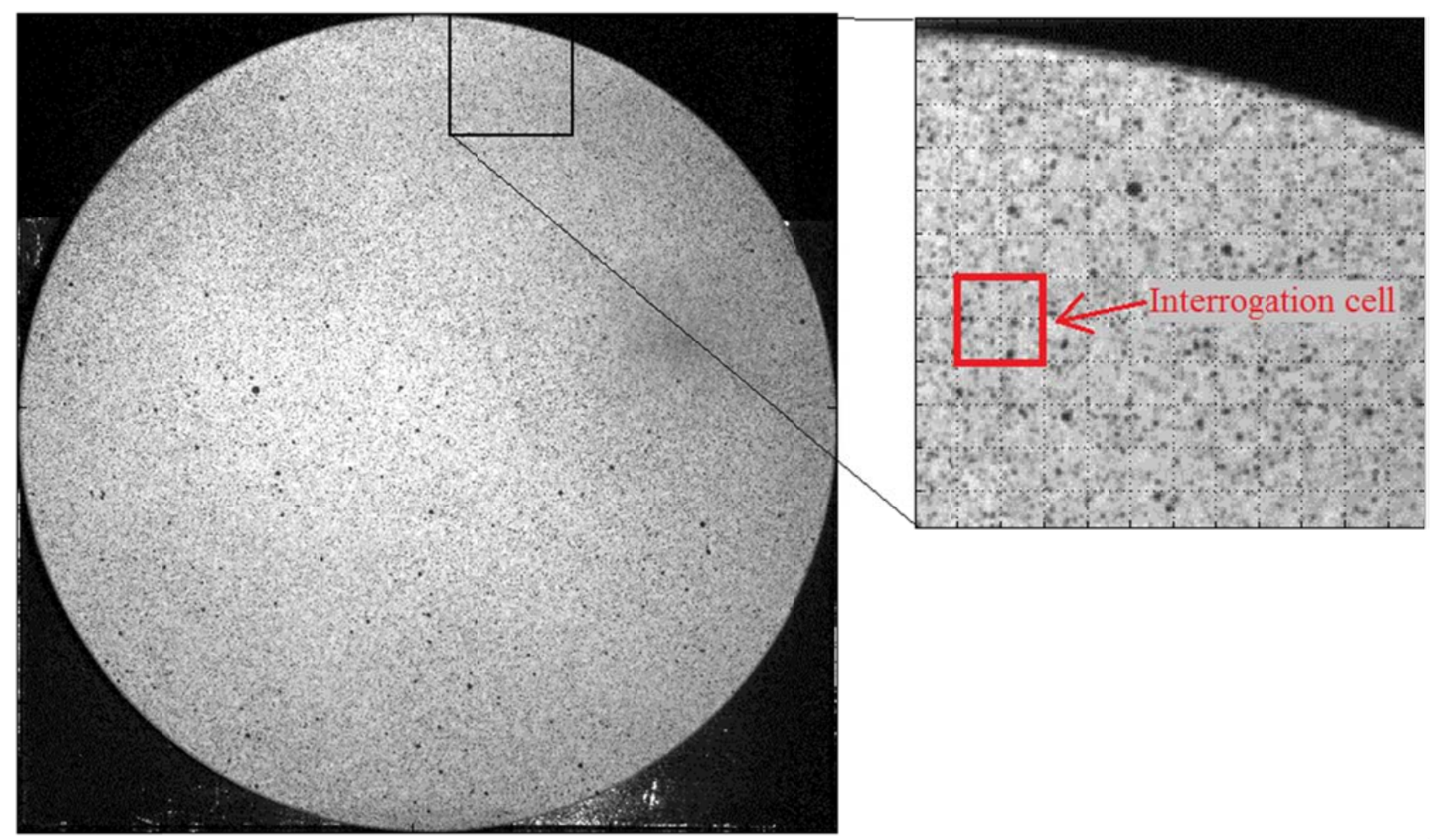

Figure 3: Example image of the disc specimen: the insert shows detail of the speckle relative to the grid of interrogation cells (21x21 pixels with a step size of 10 pixels).
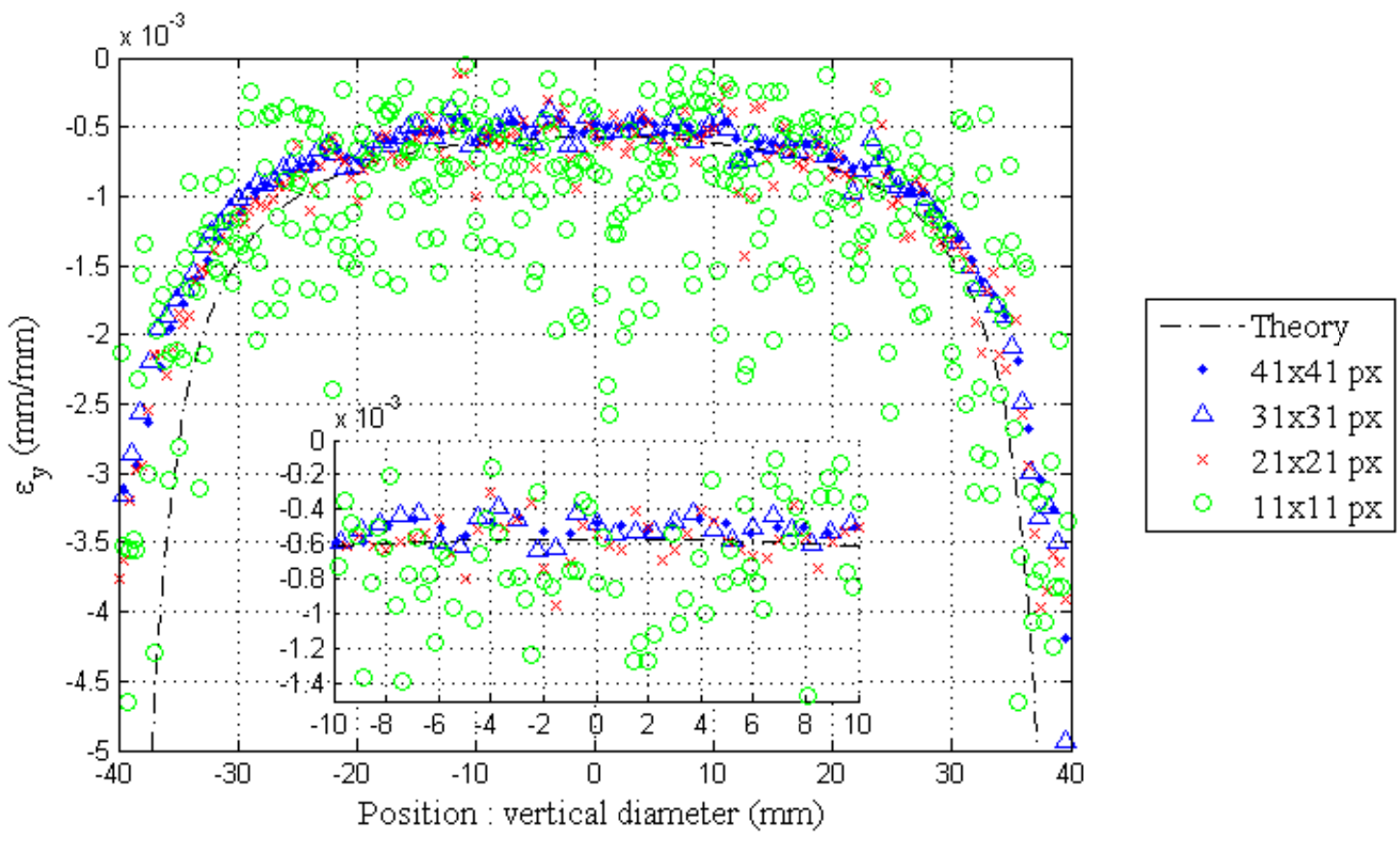

Figure 4: Comparison of $\varepsilon_{y}$ along vertical diameter calculated using different subset sizes 
Static (mean 121)

cell: $21 \mathrm{x} 21 \mathrm{px}$, step: $10 \mathrm{px}$

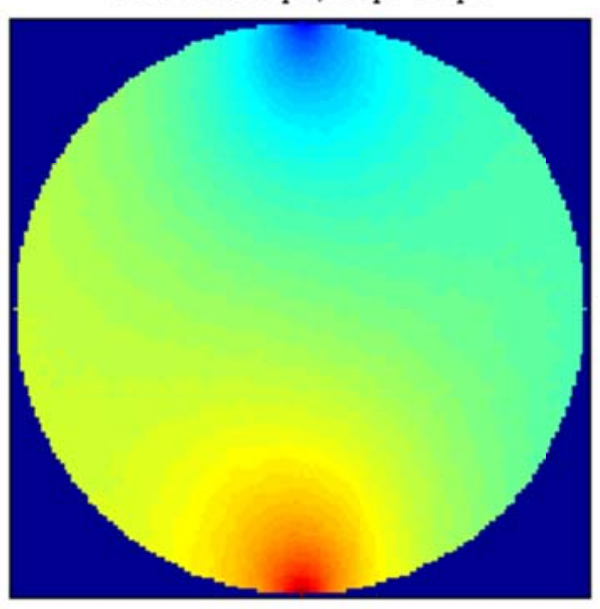

Static (mean 121) cell: $21 \mathrm{x} 21 \mathrm{px}$, step: $10 \mathrm{px}$
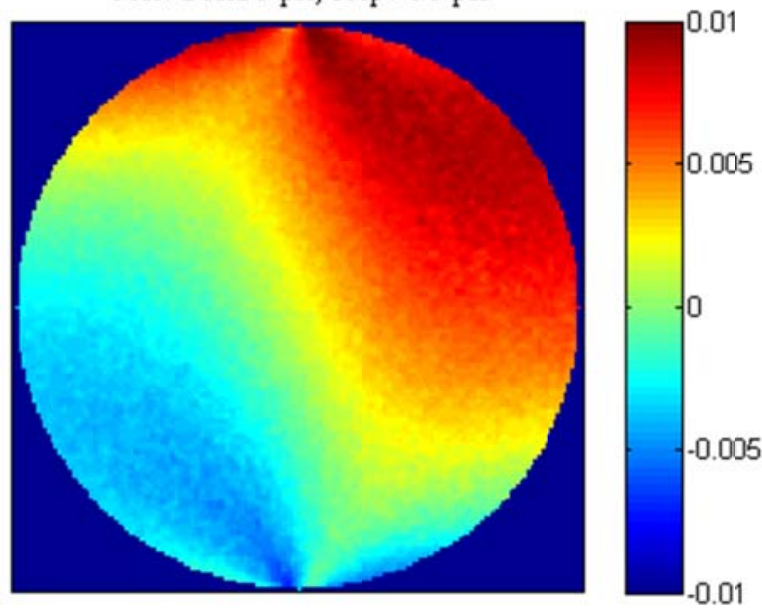

Dynamic $(0.75 \mathrm{~Hz})$

cell: $21 \mathrm{x} 21 \mathrm{px}$, step: $10 \mathrm{px}$
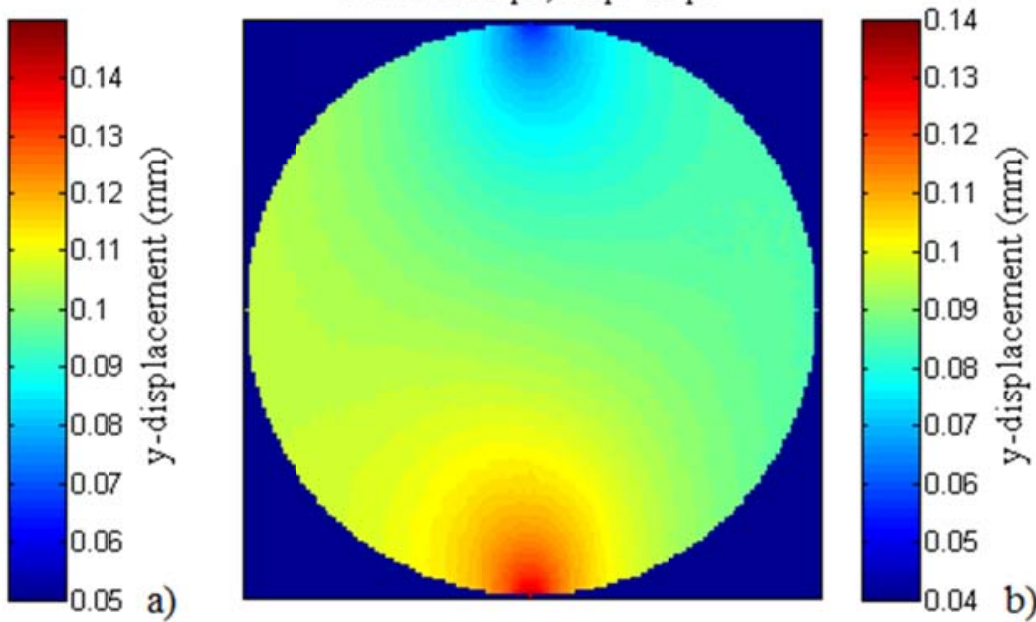

Dynamic $(0.75 \mathrm{~Hz})$ cell: $21 \mathrm{x} 21 \mathrm{px}$, step: $10 \mathrm{px}$
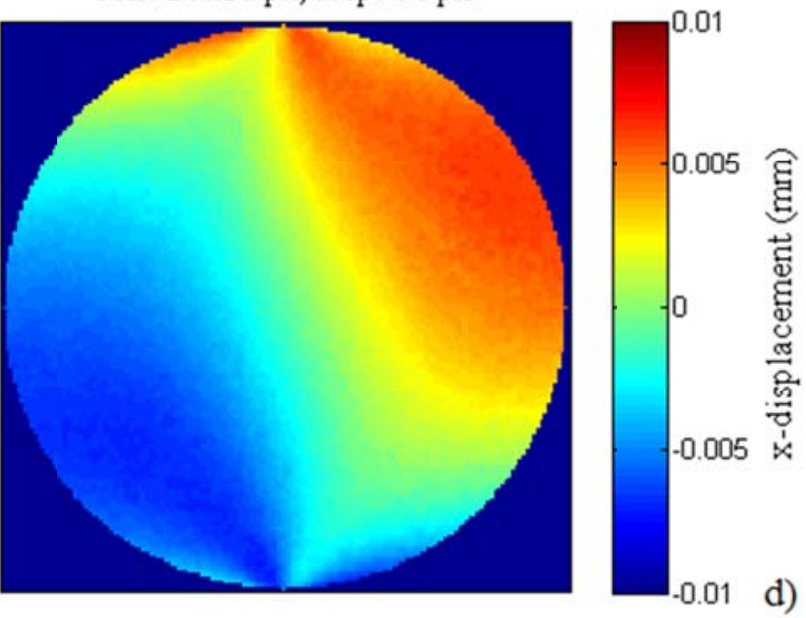

Figure 5: $y$-displacement fields from a) the static test and b) the first dynamic test at $0.75 \mathrm{~Hz}$ and the corresponding $x$-displacement fields, c) static and d) dynamic. 


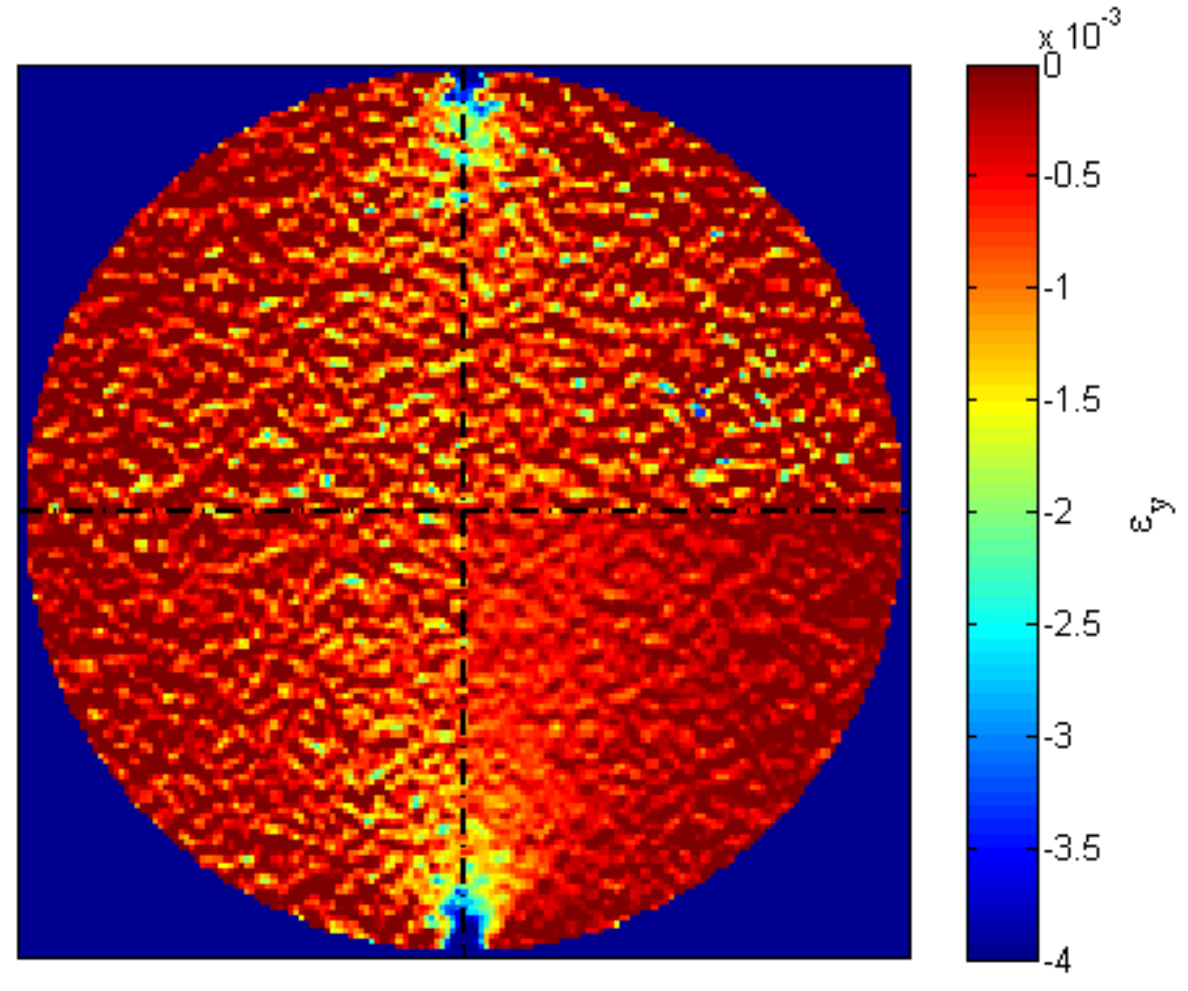

Figure 6: disc under static load, $21 \times 21$ pixel correlation subset with 11 pixel overlap, average of 1, 5, 25 and 121 strain calculations: split into quarters, top left to bottom right.

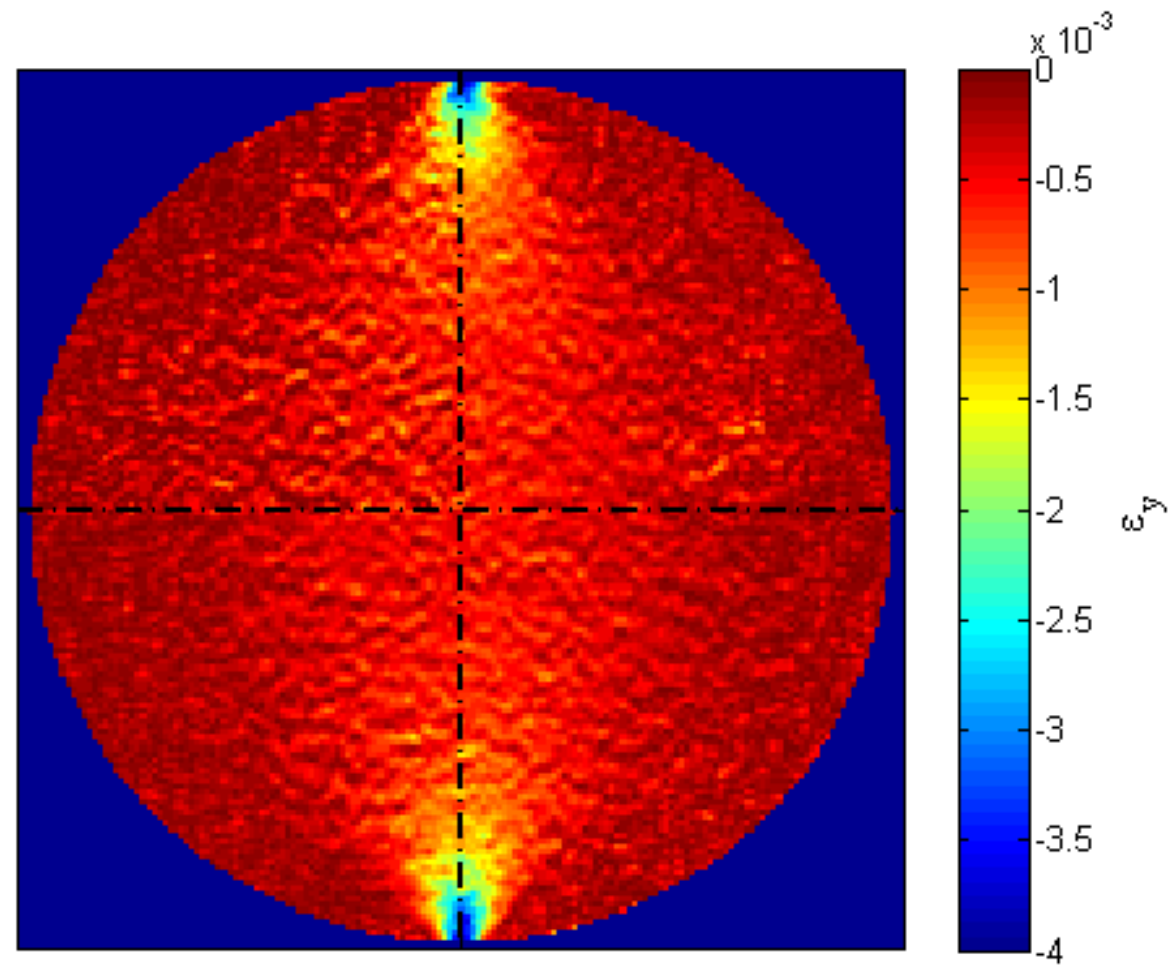

Figure 7: comparison between the disc under static load (average of 121 strain fields) and the dynamically loaded disc at $0.75 \mathrm{~Hz}, 7.1 \mathrm{~Hz}$ and $21.1 \mathrm{~Hz}$ : split into quadrants, top left to bottom right. 

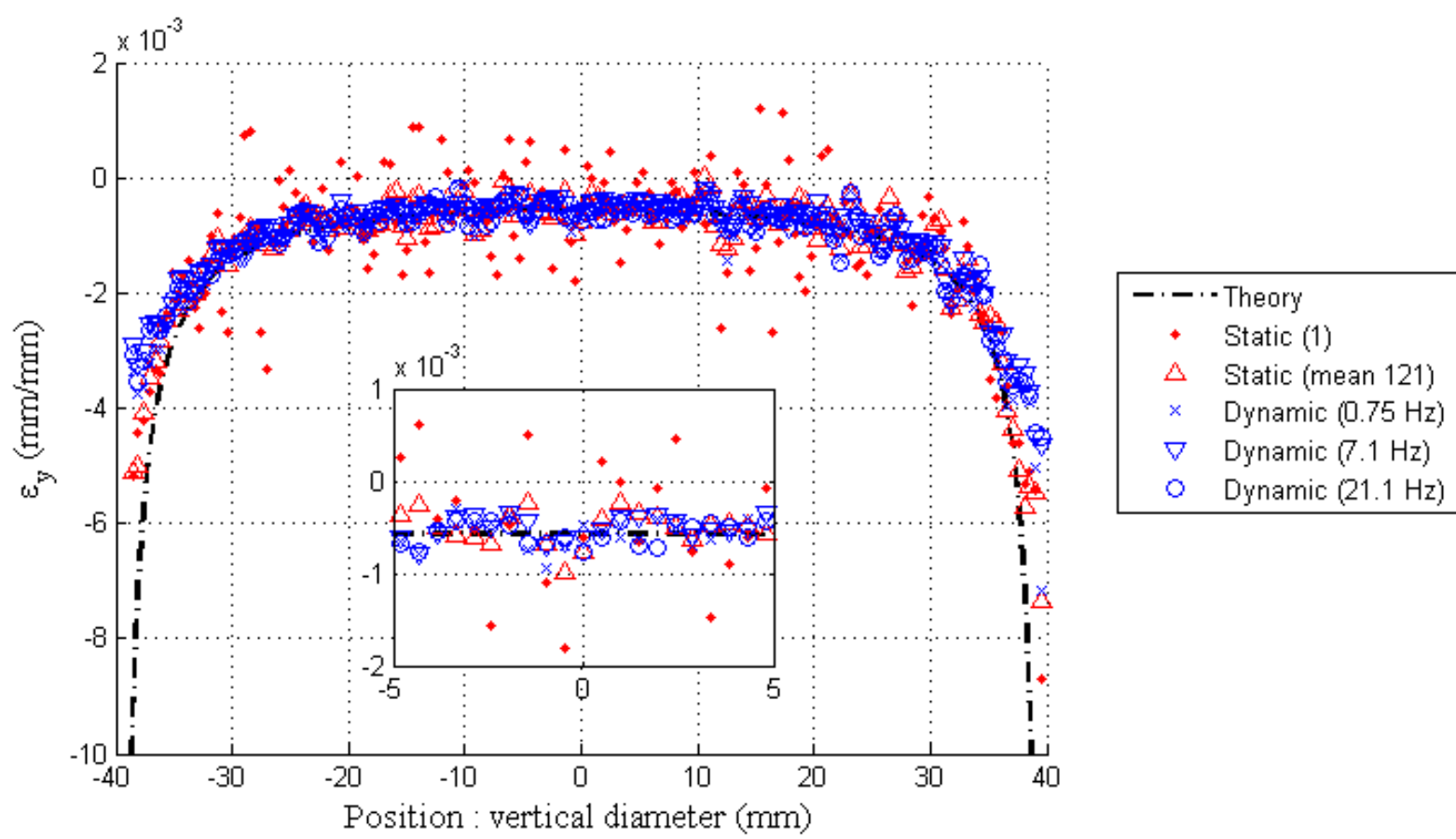

Figure 8: comparison of $\varepsilon_{y}$ along the vertical diameter obtained from the Muskhelishvili solution and the experiment. The insert shows a magnified view of the central portion of the curve.

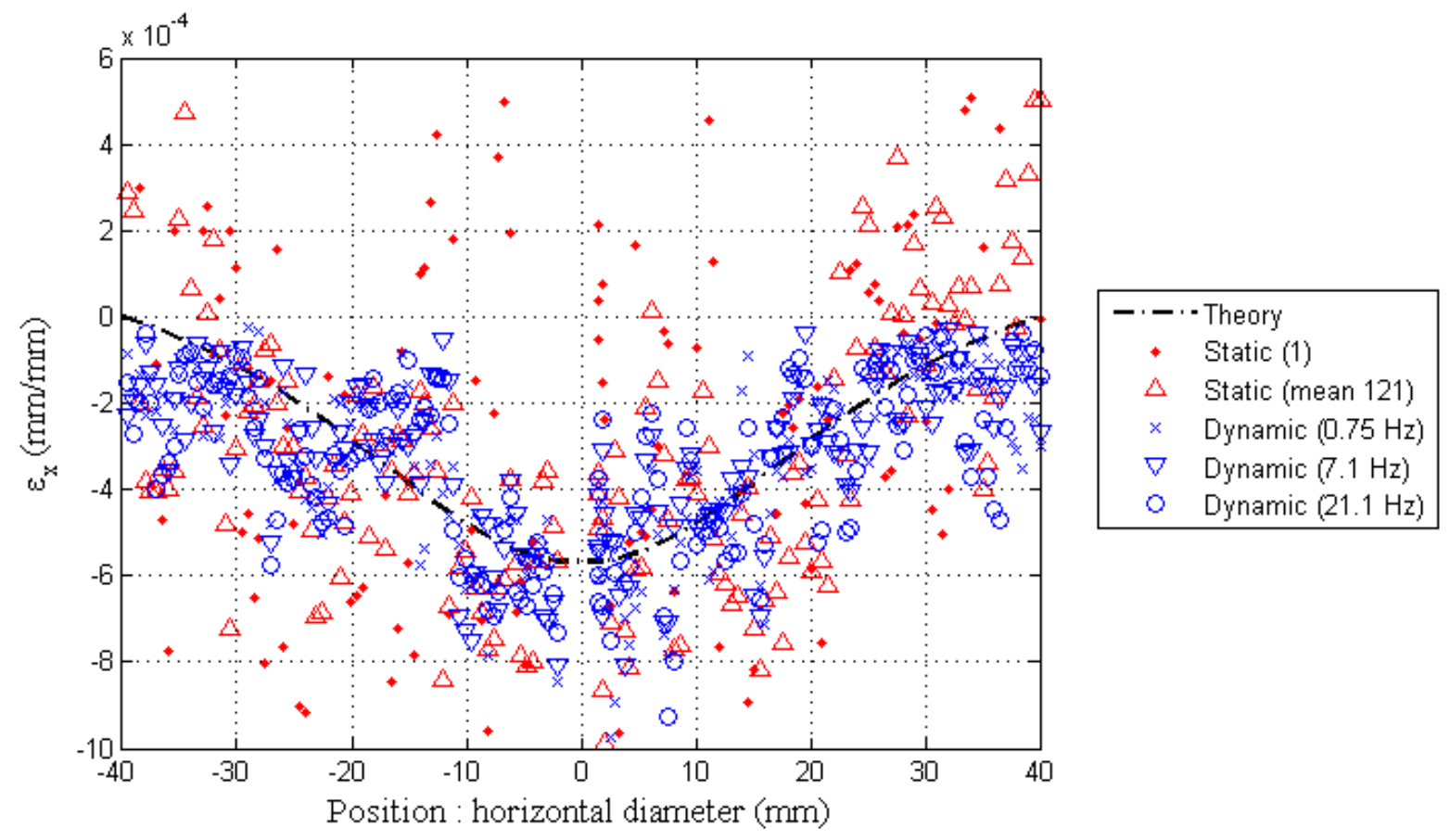

Figure 9: comparison of $\varepsilon_{y}$ along the horizontal diameter obtained from the Muskhelishvili solution and the experiment. 

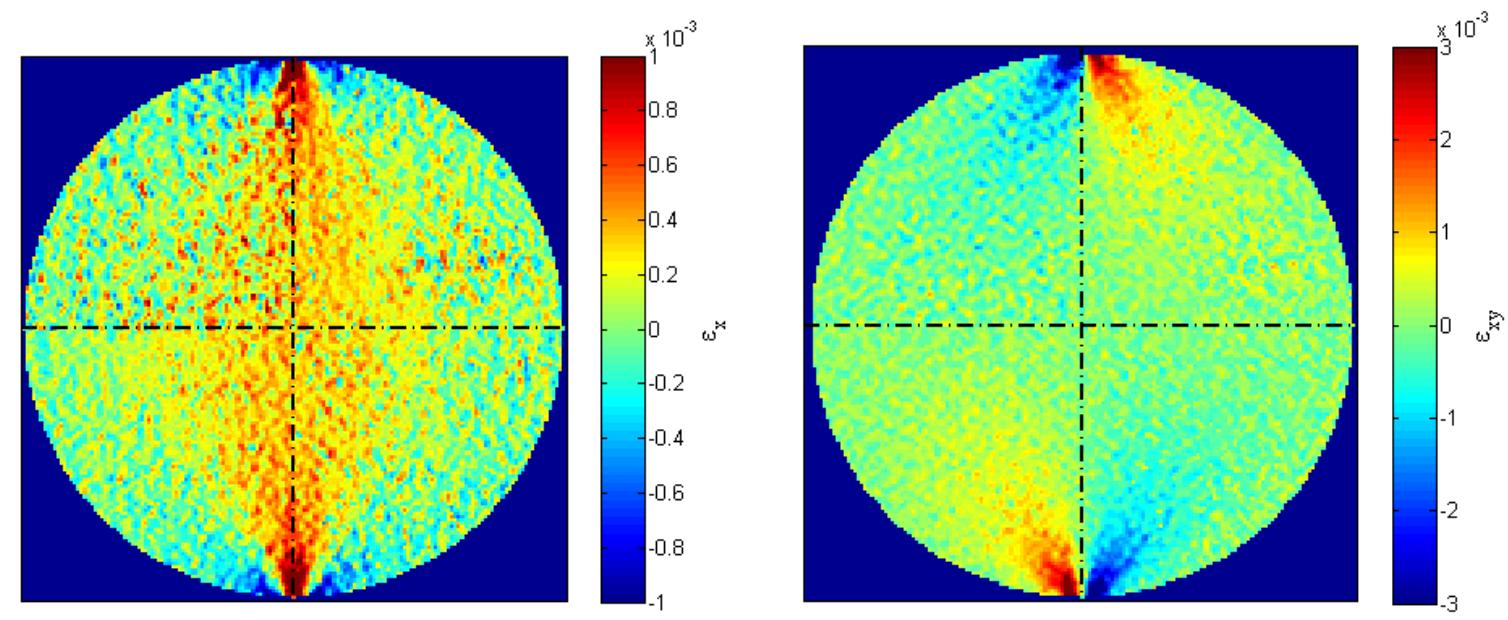

a)

b)
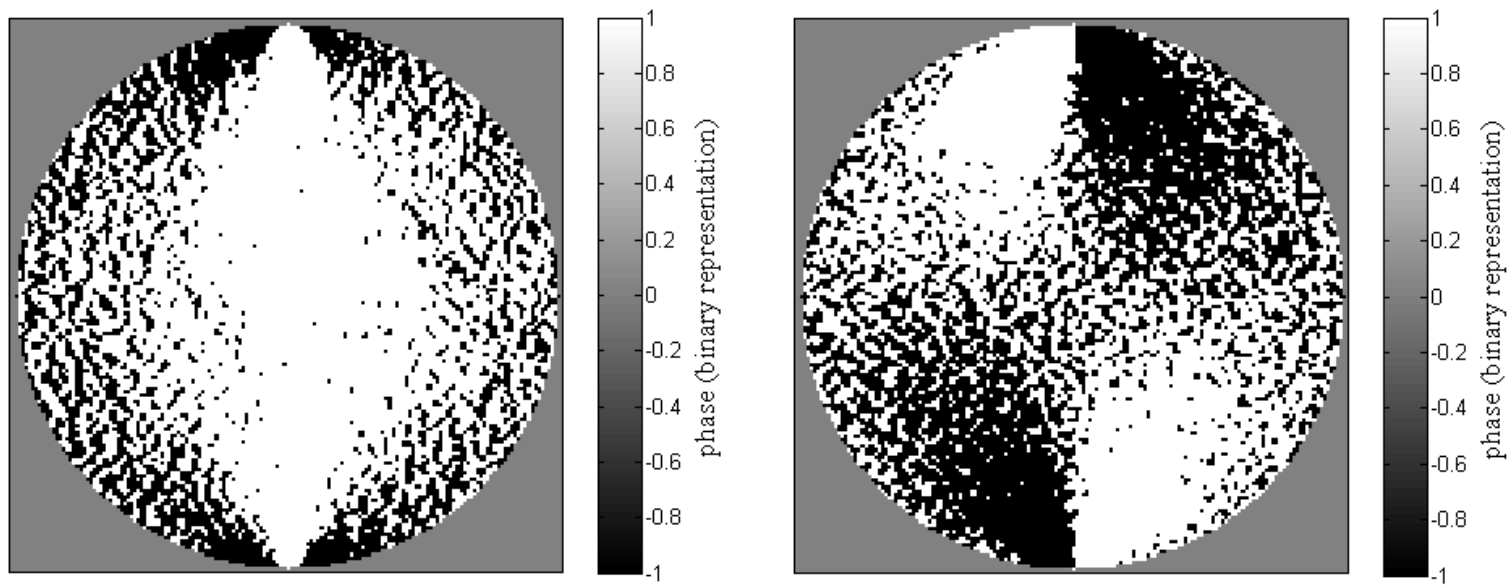

c)

d)

Figure 10: comparison of a) $\varepsilon_{x}$ and b) $\varepsilon_{x y}$ of the disc under static load (average of 121 strain fields) and the dynamically loaded disc at $0.75 \mathrm{~Hz}, 7.1 \mathrm{~Hz}$ and $21.1 \mathrm{~Hz}$ : split into quadrants, top left to bottom right. The phase data for $0.75 \mathrm{~Hz}$ is shown as a binary image $\left(1=\right.$ greater than $0^{\circ},-1=$ less than $0^{\circ}$ ) for c) $\varepsilon_{x}$ and d) $\varepsilon_{x y}$. 


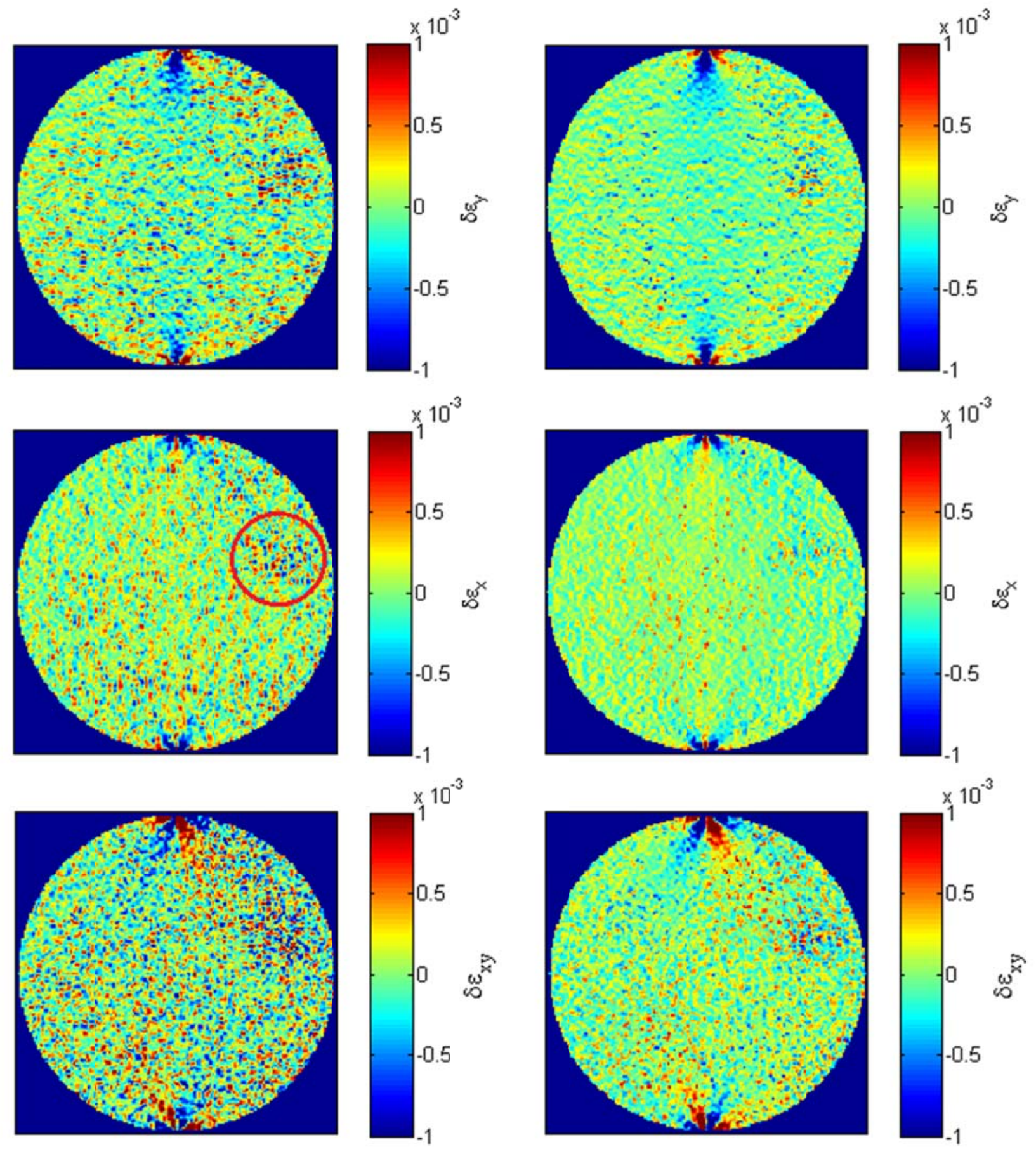

Figure 11: Images of the difference between the theoretical strains and the experimentally determined strains for the static mean of 121 images and the $0.75 \mathrm{~Hz}$ dynamic cases. 


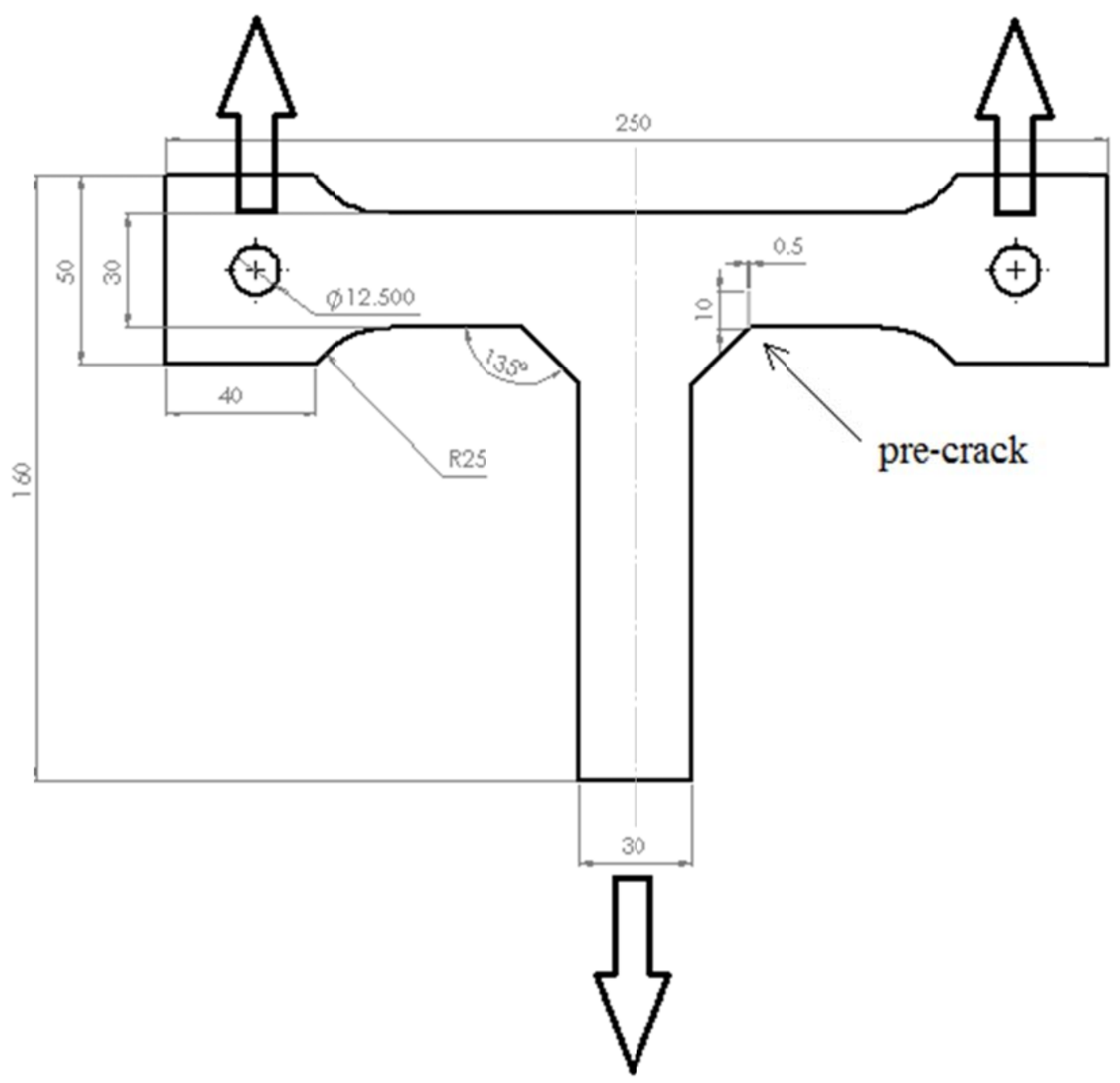

Figure 12: T-specimen with pre-crack: dimensions and mode of loading. 


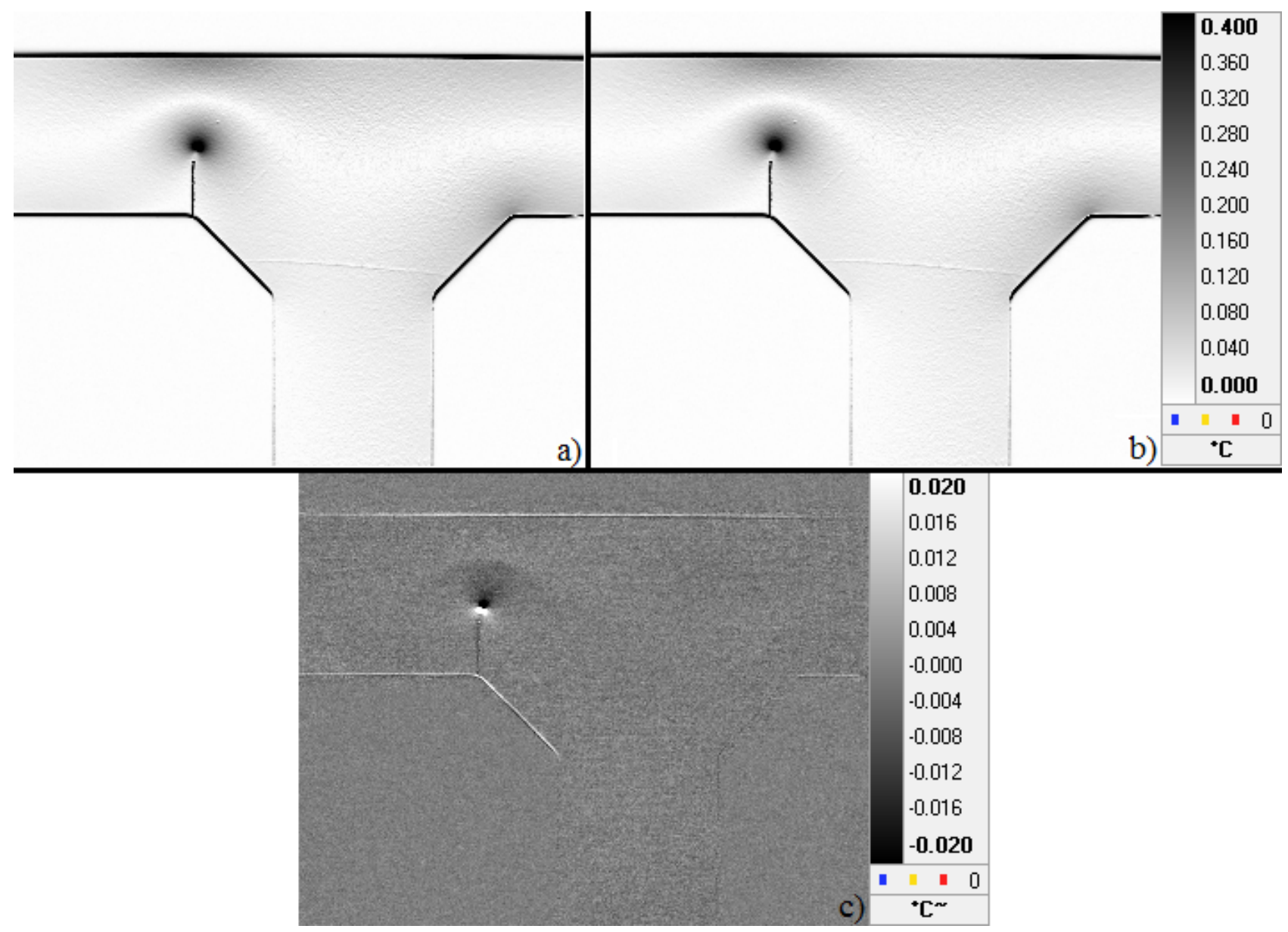

Figure 13: Comparison of TSA data at the start and end of white light image recording at 100,000 cycles: a) $\Delta T$ at 100,000 cycles, b) $\Delta T$ at 102,000 cycles, c) difference $\left(\Delta T_{100}-\Delta T_{102}\right)$. 


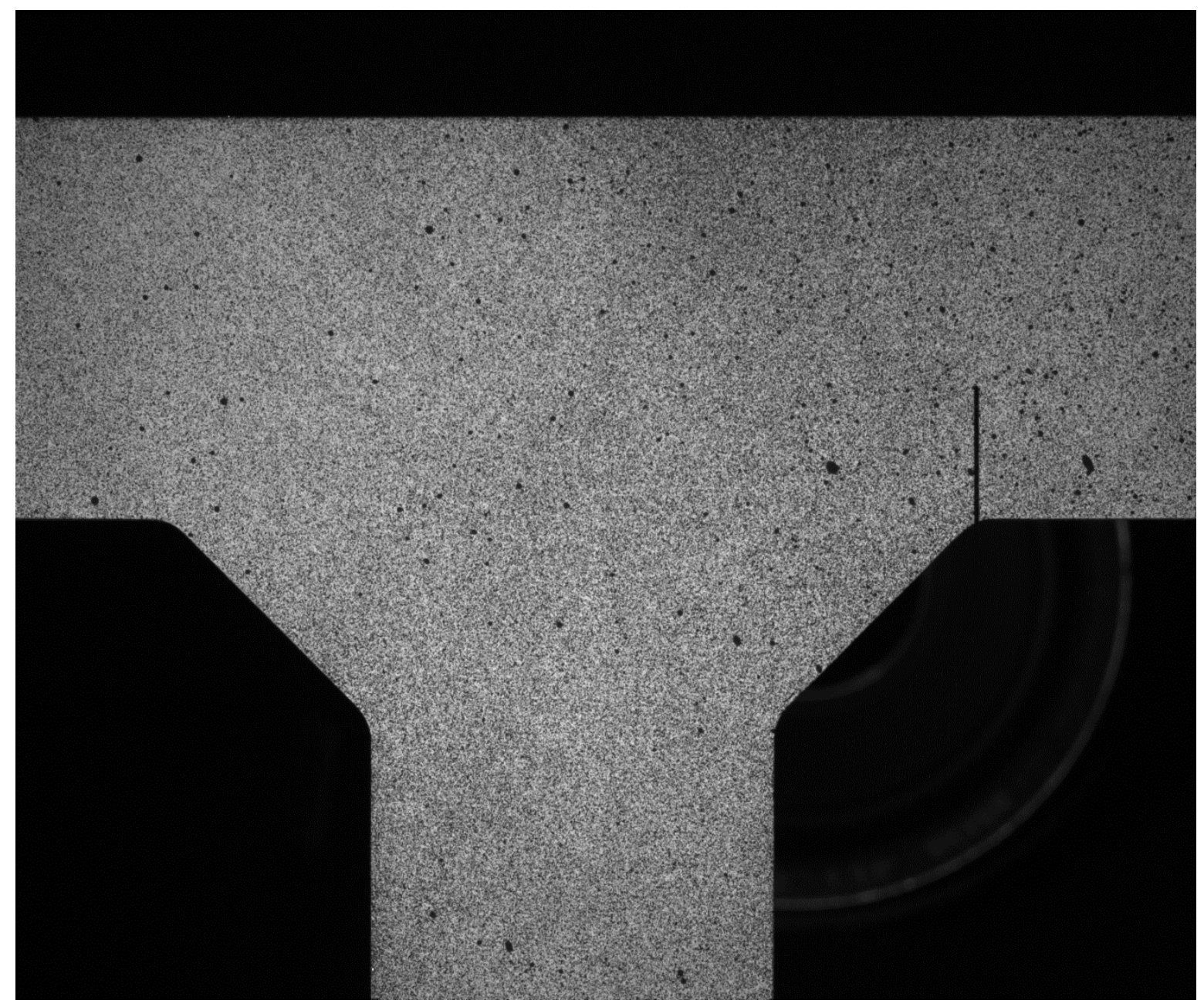

Figure 14: Example image showing the speckle applied to the T-shaped specimen. Note: the lens of the infrared camera can be seen in the background. 
0 cycles

subset $=21 \mathrm{x} 21 \mathrm{px}$, step $=5 \mathrm{px}$

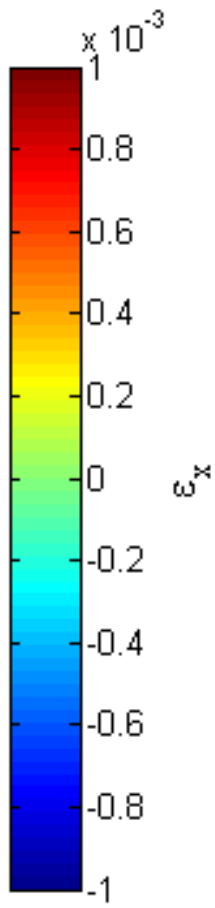

100,000 cycles

subset $=21 \mathrm{x} 21 \mathrm{px}$, step $=5 \mathrm{px}$
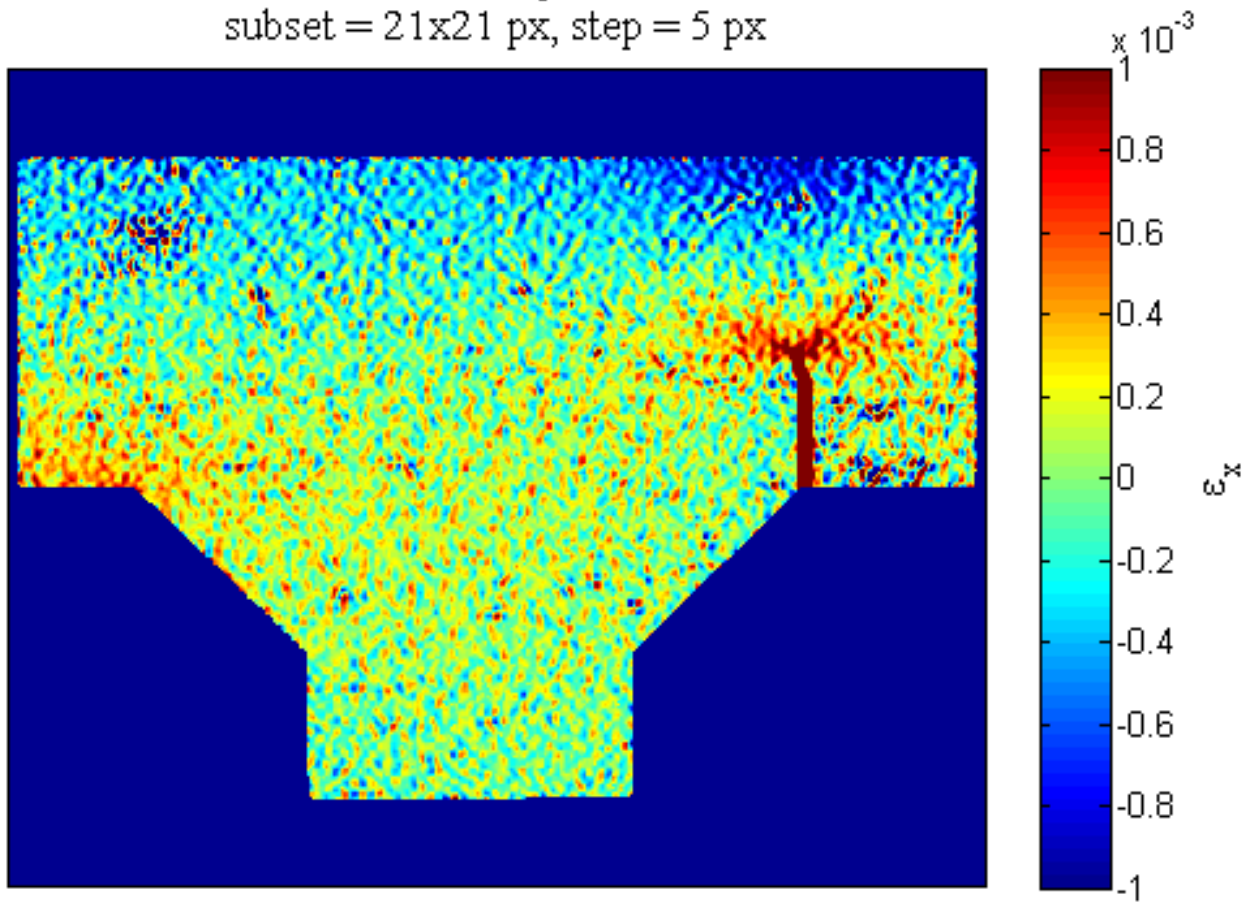

Figure 15: $\varepsilon_{x}$ strain fields using a subset size of $21 \times 21$ px (step size 5 px) 

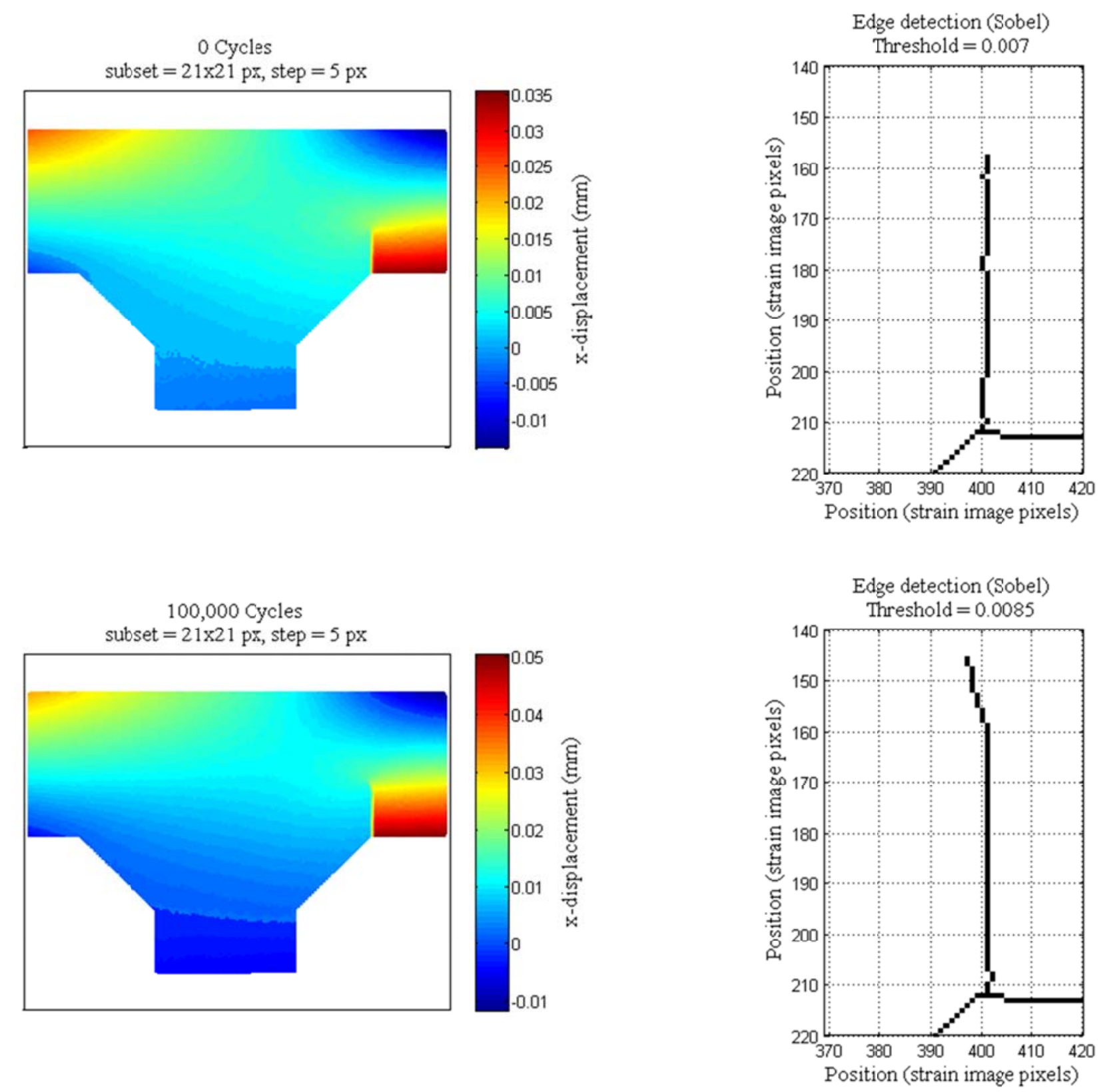

Figure 16; $x$-displacement fields (21x21 px subset with 5 px step size) and corresponding crack identified using Matlab's 'edge' function employing the 'Sobel' algorithm. 


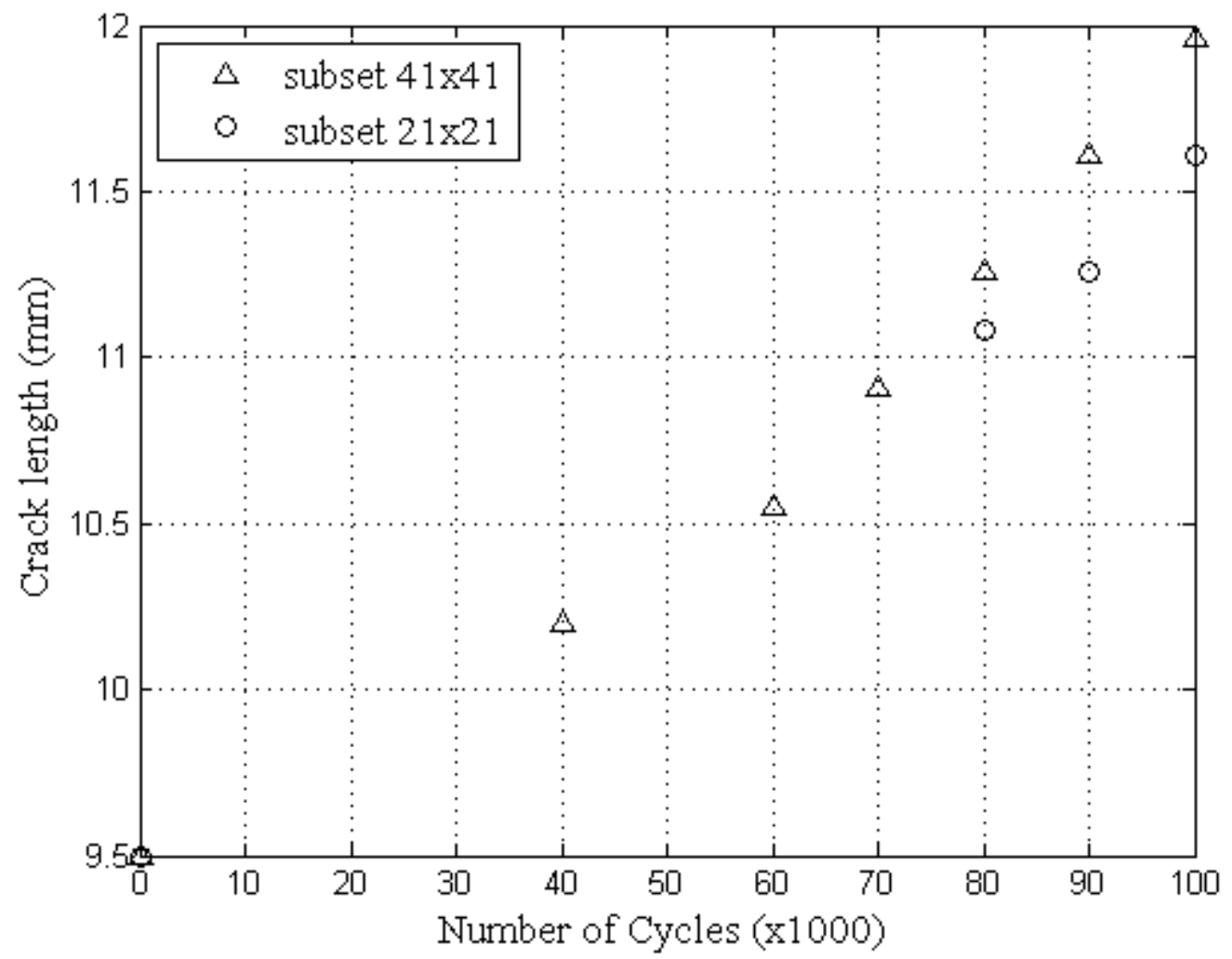

Figure 17: Change in crack length with number of cycles, established using the Matlab 'edge' function with the 'Sobel' algorithm. 